TRANSACTIONS OF THE

AMERICAN MATHEMATICAL SOCIETY

Volume 358, Number 3, Pages 1223-1255

S 0002-9947(05)03744-X

Article electronically published on May 9, 2005

\title{
BROWNIAN INTERSECTION LOCAL TIMES: EXPONENTIAL MOMENTS AND LAW OF LARGE MASSES
}

\author{
WOLFGANG KÖNIG AND PETER MÖRTERS
}

\begin{abstract}
Consider $p$ independent Brownian motions in $\mathbb{R}^{d}$, each running up to its first exit time from an open domain $B$, and their intersection local time $\ell$ as a measure on $B$. We give a sharp criterion for the finiteness of exponential moments,

$$
\mathbb{E}\left[\exp \left(\sum_{i=1}^{n}\left\langle\varphi_{i}, \ell\right\rangle^{1 / p}\right)\right]
$$

where $\varphi_{1}, \ldots, \varphi_{n}$ are nonnegative, bounded functions with compact support in $B$. We also derive a law of large numbers for intersection local time conditioned to have large total mass.
\end{abstract}

\section{Introduction AND STATEMENT OF RESUlts}

1.1. Introduction. Much of our knowledge about Brownian occupation times goes back to the celebrated Feynman-Kac formula; see e.g. [Fe48, Ka49] for original sources and [FP99 for an excellent survey. The formula is also at the heart of results relating Brownian motion and a vast number of differential equations; see BS02. for an impressive account. To formulate one of many versions of the Feynman-Kac formula, let $B \subset \mathbb{R}^{d}$ be a bounded open domain and suppose that $W$ is a Brownian motion started in $x \in B$. Let $q: B \rightarrow[0, \infty)$ be a Borel measurable function and let $T$ be the first exit time of the Brownian motion $W$ from $B$. Then the function $f: B \rightarrow[0, \infty]$ given by

$$
f(x)=\mathbb{E}_{x}\left[\exp \int_{0}^{T} q(W(s)) d s\right], \text { for } x \in B,
$$

is the minimal positive solution of the equation

$$
f(x)=1+\int G(x, y) f(y) q(y) d y
$$

where $G$ is the Green function for Brownian motion killed on the boundary of $B$; see e.g. [FP99, (8)]. Hence, the exponential moments in (1.1) are finite if and only if there exists a finite positive solution to (1.2).

An explicit criterion for the finiteness of the exponential moments in (1.1), given in terms of a variational formula, is due to Pinsky [Pi86]. Assuming that $B$ is a

Received by the editors August 13, 2003 and, in revised form, May 4, 2004.

2000 Mathematics Subject Classification. Primary 60J65, 60J55, 60F10.

Key words and phrases. Intersection of Brownian paths, intersection local time, exponential moment, Feynman-Kac formula.

(C)2005 American Mathematical Society Reverts to public domain 28 years from publication 
bounded, open domain with a $C^{2}$-boundary, for any continuous function $q: \bar{B} \rightarrow \mathbb{R}$, define

$$
l_{q, B}=\sup \left\{\int q(x) \psi(x)^{2} d x-\frac{1}{2}\|\nabla \psi\|_{2}^{2}: \psi \in H_{0}^{1}(B), \int \psi(x)^{2} d x=1\right\} .
$$

Pinsky shows that

$$
\mathbb{E}_{x}\left[\exp \int_{0}^{T} q(W(s)) d s\right] \quad\left\{\begin{array}{l}
<\infty \text { for all } x \in B \text { if } l_{q, B}<0 \\
=\infty \text { for all } x \in B \text { if } l_{q, B}>0
\end{array}\right.
$$

The heuristics behind this formula is that the main contribution to the exponential moment comes from Brownian motion behaving as if under the influence of a drift field $\nabla \psi / \psi$ for a large time $t \rightarrow \infty$, where $\psi$ is a maximiser in (1.3). The cost of following this drift is given by $\exp \left(-t \frac{1}{2}\|\nabla \psi\|_{2}^{2}\right)$, and the exponent itself becomes $t \int q(x) \psi(x)^{2} d x$.

Whereas we have an almost complete understanding of occupation times of one Brownian path, we know much less about the intersection of several independent Brownian paths, where the role of occupation times is played by intersection local times. However, there is every indication that the relation of intersection local times and differential equations is as rich and exciting as in the case of a single Brownian motion. Maybe even more so, as the equations appearing in this context turn out to be nonlinear, and the analytical theory of these equations knows more open problems than answers.

The crucial tools for the study of occupation measures, the Feynman-Kac formula and the Donsker-Varadhan large deviation theory, can still be used in the analysis of intersection local times; see Ch04 for a skillful example. However, in order to improve our understanding of intersection local times, it would be very important to have natural analogues for these results which apply directly to intersection local times. In this paper, as a first step into this new territory, we investigate the existence of exponential moments for intersection local times of $p$ Brownian motions in $\mathbb{R}^{d}$. In analogy to (1.4) we give a finiteness criterion for exponential moments of integrals of intersection local time against a large class of test functions. In the absence of the two crucial tools mentioned before, our arguments rely heavily on combinatorial and analytical methods. As a consequence of our approach, we are able to prove a law of large numbers (or, more accurately, large masses) which relates the asymptotic shape of intersection local time to a natural nonlinear partial differential equation.

1.2. Brownian intersection local times. Suppose that $B \subset \mathbb{R}^{d}$, with $d \geq 1$, is an open set. The set $B$ is assumed to be bounded if $d \leq 2$, except that we allow $B=\mathbb{R}^{d}$, if $d \geq 3$. Let $p \geq 1$ be an integer, $x=\left(x_{1}, \ldots, x_{p}\right) \in B^{p}$, and assume that a family of $p$ independent Brownian motions

$$
\left(W^{1}(t): t \in[0, \infty)\right), \ldots,\left(W^{p}(t): t \in[0, \infty)\right)
$$

in $\mathbb{R}^{d}$ with $W^{1}(0)=x_{1}, \ldots, W^{p}(0)=x_{p}$ are realized on a probability space $\left(\Omega, \mathcal{F}, \mathbb{P}_{x}\right)$. Denote the corresponding expectation by $\mathbb{E}_{x}$. Each motion is killed at the first exit time $T^{i}=\inf \left\{t>0: W^{i}(t) \notin B\right\}$ from $B$ if this time is finite. By classical results of Dvoretzky, Erdős, Kakutani and Taylor, $p(d-2)<d$ is equivalent to the fact that the paths of the $p$ motions have a positive probability of intersecting in a point other than their starting point. In this case there exists a 
locally finite measure, the (projected) intersection local time measure $\ell$, which can symbolically be described by the formula

$$
\ell(A)=\int_{A} d y \prod_{i=1}^{p} \int_{0}^{T^{i}} d s \delta_{y}\left(W^{i}(s)\right), \text { for } A \subset \mathbb{R}^{d} \text { Borel. }
$$

Heuristically, $\ell(A)$ measures the amount of intersection of the $p$ Brownian paths in the set $A$ before they are killed. The measure $\ell$ is a random element of the space $\mathcal{M}(B)$ of nonnegative, locally finite measures on $B$, which is equipped with the vague topology. $\ell$ is nontrivial with positive probability and, if $A$ is a bounded set, $\ell(A)$ is almost surely finite. If $A$ is unbounded, then $\ell(A)$ may be equal to $\infty$ with positive probability.

We always assume that $p(d-2)<d$. This includes the following cases:

- $p=1, d$ arbitrary. In this case $\ell$ degenerates to the occupation measure of a single Brownian path,

$$
\ell(A)=\int_{0}^{T^{1}} d s 1_{A}\left(W^{1}(s)\right), \text { for } A \subset \mathbb{R}^{d} \text { Borel } .
$$

Our main result, Theorem 1.1, is essentially contained in [Pi86]; see Remark 1.4 for a comparison. Theorem 1.6 seems to be new even in this case.

- $d=1, p \geq 2$ arbitrary. In this case the symbolic formula (1.5) makes sense using local time. Indeed, if $\left(L^{i}(x): x \in \mathbb{R}\right)$ is the family of local times of the stopped Brownian motion $W^{i}$, i.e., the continuous density of the occupation measure $\int_{0}^{T^{i}} d s \delta_{W^{i}(s)}$, we define

$$
\ell(A)=\int_{A} d y \prod_{i=1}^{p} L^{i}(y), \text { for } A \subset \mathbb{R} \text { Borel. }
$$

- $d=2, p \geq 2$ arbitrary, and $d=3, p=2$. In these most interesting cases, the local times do not exist, and substantial work is needed to turn (1.5) into a rigorous definition. See Section 2 of KM02 for a short survey on three rigorous constructions of $\ell$ in these cases.

We would like to mention (see LG87, LG89]) that if $d \geq 2$, almost surely, $\ell$ is equal to a Hausdorff measure on the set $S:=W^{1}\left[0, T^{1}\right) \cap \ldots \cap W^{p}\left[0, T^{p}\right)$ with some deterministic gauge function. This fact underlines that $\ell$ is the natural measure on the intersection of the paths.

1.3. The main result. Let $\varphi: B \rightarrow[0, \infty)$ be bounded with compact support in $B$. If $\mu$ is a measure on $B$ we write $\langle\varphi, \mu\rangle=\int \varphi d \mu$. Suppose now that $\varphi$ is positive on a set of positive Lebesgue measure; then it turns out that $\mathbb{E}_{x}[\exp (\langle\varphi, \ell\rangle)]=\infty$. However, it is a subtle question whether the stretched exponential moments of the form

$$
\mathbb{E}_{x}\left[\exp \left(\langle\varphi, \ell\rangle^{1 / p}\right)\right]
$$

where $p$ is the number of Brownian motions, are finite or not. Our first main result is a sharp criterion for this. In fact, the nonlinearity due to the $p$ th root in the exponent makes it natural to ask a more general question, namely when for a finite 
family $\left(\varphi_{1}, \ldots, \varphi_{n}\right)$ of bounded nonnegative functions the moments

$$
\mathbb{E}_{x}\left[\exp \left(\sum_{i=1}^{n}\left\langle\varphi_{i}, \ell\right\rangle^{1 / p}\right)\right]
$$

are infinite or not. To formulate our answer denote by

$$
\mathcal{D}(B)= \begin{cases}H_{0}^{1}(B) & \text { if } B \text { is bounded } \\ D^{1}\left(\mathbb{R}^{d}\right) & \text { if } B=\mathbb{R}^{d}\end{cases}
$$

the classical Sobolev space $H_{0}^{1}(B)$ with zero boundary condition if $B$ is bounded, and, in the case that $B=\mathbb{R}^{d}$, the set $D^{1}\left(\mathbb{R}^{d}\right)$ of functions in $L_{\mathrm{loc}}^{1}\left(\mathbb{R}^{d}\right)$ vanishing at infinity and having a distributional gradient in $L^{2}\left(\mathbb{R}^{d}\right)$. In Section 5 we recall some properties of $\mathcal{D}(B)$.

Theorem 1.1 (Exponential moments). Let $\phi=\left(\phi_{1}, \ldots, \phi_{n}\right)$ be a family of bounded nonnegative Borel measurable functions with compact support in $B$, and let

$$
\Theta(\phi)=\Theta\left(\phi_{1}, \ldots, \phi_{n}\right)=\inf \left\{\frac{p}{2}\|\nabla \psi\|_{2}^{2}: \psi \in \mathcal{D}(B), \sum_{i=1}^{n}\left\|\phi_{i} \psi\right\|_{2 p}^{2}=1\right\} .
$$

Then

$$
\mathbb{E}_{x}\left[\exp \left(\sum_{i=1}^{n}\left\langle\phi_{i}^{2 p}, \ell\right\rangle^{1 / p}\right)\right]\left\{\begin{array}{l}
<\infty \text { for all } x \in B^{p} \text { if } \Theta(\phi)>1, \\
=\infty \text { for all } x \in B^{p} \text { if } \Theta(\phi)<1 .
\end{array}\right.
$$

Indeed, we even have

$$
\lim _{a \uparrow \infty} \frac{1}{a} \log \mathbb{P}_{x}\left\{\sum_{i=1}^{n}\left\langle\phi_{i}^{2 p}, \ell\right\rangle^{1 / p}>a\right\}=-\Theta(\phi) .
$$

A partial result in the direction of Theorem 1.1 was obtained in KM02. From Theorem 1.1 we can infer a finiteness criterion for intersection local times in a form analogous to Pinsky's result for single Brownian motion in (1.4).

Corollary 1.2. Let $B \subset \mathbb{R}^{d}$ be a bounded, open domain with $C^{1}$-boundary, and let $\phi=\left(\phi_{1}, \ldots, \phi_{n}\right)$ be a family of bounded Borel measurable functions $\phi_{i}: \bar{B} \rightarrow[0, \infty)$. Let

$$
l_{\phi, B}^{p}=\sup \left\{\sum_{i=1}^{n}\left\|\phi_{i} \psi\right\|_{2 p}^{2}-\frac{p}{2}\|\nabla \psi\|_{2}^{2}: \psi \in \mathcal{D}(B),\|\psi\|_{2 p}=1\right\} .
$$

Then

$$
\mathbb{E}_{x}\left[\exp \left(\sum_{i=1}^{n}\left\langle\phi_{i}^{2 p}, \ell\right\rangle^{1 / p}\right)\right]\left\{\begin{array}{l}
<\infty \text { for all } x \in B^{p} \text { if } l_{\phi, B}^{p}<0 \\
=\infty \text { for all } x \in B^{p} \text { if } l_{\phi, B}^{p}>0
\end{array}\right.
$$

Remark 1.3. As in Pinsky's result, heuristically, the main contribution to the exponential moment comes from each Brownian motion behaving as if under the influence of a drift field $\nabla \psi / \psi$ for $t \rightarrow \infty$, which pushes the Brownian motion towards the origin and leads to occupation densities $t \psi(x)^{2}$ for each individual path, and thus $t^{p} \psi(x)^{2 p}$ for the intersections. In line with Donsker-Varadhan theory, the cost to realize these occupation densities for all $p$ motions is given by $\exp \left(-t \frac{1}{2}\|\nabla \psi\|_{2}^{2}\right)$ for each motion, and the exponent itself becomes $t \sum_{i=1}^{n}\left\|\phi_{i} \psi\right\|_{2 p}^{2}$. 
Proof. For $\varepsilon>0$, denote by $B[\varepsilon]$ the open $\varepsilon$-neighbourhood of $B$. We first show that

$$
\limsup _{\varepsilon \downarrow 0} l_{\phi, B[\varepsilon]}^{p} \leq l_{\phi, B}^{p} .
$$

Indeed, for $k \in \mathbb{N}$ let $\psi_{k} \in H_{0}^{1}(B[1 / k])$ be an approximate minimizer in (1.10) i.e., $\left\|\psi_{k}\right\|_{2 p}=1$ and $\sum_{i=1}^{n}\left\|\phi_{i} \psi_{k}\right\|_{2 p}^{2}-\frac{p}{2}\left\|\nabla \psi_{k}\right\|_{2}^{2} \geq l_{\phi, B[1 / k]}^{p}-1 / k$. Since the first term is bounded in $k \in \mathbb{N}$, it is clear that $\left(\left\|\nabla \psi_{k}\right\|^{2}\right)_{k \in \mathbb{N}}$ is bounded. By Lemma 5.2 we may assume that $\psi_{k}$ converges, as $k \rightarrow \infty$, to some $\psi \in H_{0}^{1}(B[1])$ in $L^{2 p_{-}}$ norm such that $\nabla \psi_{k}$ converges weakly to $\nabla \psi$. Since $\operatorname{supp}\left(\psi_{k}\right) \subset B[1 / k]$ for any $k \in \mathbb{N}$, we may assume that $\psi \in H^{1}\left(\mathbb{R}^{d}\right)$ with $\psi=0$ outside $B$. According to Lemma 5.1. the restriction of $\psi$ to $B$ lies in $H_{0}^{1}(B)$. By lower semicontinuity of $\|\cdot\|_{2}$, we have $\|\nabla \psi\|_{2} \leq \liminf _{k \uparrow \infty}\left\|\nabla \psi_{k}\right\|_{2}$. By $L^{2 p}$-convergence we have $\lim _{k \uparrow \infty} \sum_{i=1}^{n}\left\|\phi_{i} \psi_{k}\right\|_{2 p}^{2}=\sum_{i=1}^{n}\left\|\phi_{i} \psi\right\|_{2 p}^{2}$. Hence,

$$
\limsup _{\varepsilon \downarrow 0} l_{\phi, B[\varepsilon]}^{p} \leq \sum_{i=1}^{n}\left\|\phi_{i} \psi\right\|_{2 p}^{2}-\frac{p}{2}\|\nabla \psi\|_{2}^{2} \leq l_{\phi, B}^{p}
$$

and this finishes the proof of (1.12).

Now assume that $l_{\phi, B}^{p}<0$. Because of (1.12) one can fix $\varepsilon>0$ such that $l_{\phi, B[\varepsilon]}^{p}<0$. We now work in the domain $B[\varepsilon]$ and exploit that the supports of $\phi_{1}, \ldots, \phi_{n}$ are strictly inside $B[\varepsilon]$. For any $\eta>0$,

$$
\begin{aligned}
0<-l_{\phi, B[\varepsilon]}^{p} & =\inf _{\psi \in \mathcal{D}(B[\varepsilon]) \backslash\{0\}} \frac{\frac{p}{2}\|\nabla \psi\|_{2}^{2}-\sum_{i=1}^{n}\left\|\phi_{i} \psi\right\|_{2 p}^{2}}{\|\psi\|_{2 p}^{2}} \\
& \leq \inf \left\{\frac{\frac{p}{2}\|\nabla \psi\|_{2}^{2}-1}{\|\psi\|_{2 p}^{2}}: \psi \in \mathcal{D}(B[\varepsilon]), \sum_{i=1}^{n}\left\|\phi_{i} \psi\right\|_{2 p}^{2}=1,\|\psi\|_{2 p}^{2} \geq \eta\right\} \\
& \leq \frac{1}{\eta}\left(\inf \left\{\frac{p}{2}\|\nabla \psi\|_{2}^{2}: \psi \in \mathcal{D}(B[\varepsilon]), \sum_{i=1}^{n}\left\|\phi_{i} \psi\right\|_{2 p}^{2}=1,\|\psi\|_{2 p}^{2} \geq \eta\right\}-1\right) .
\end{aligned}
$$

Since there is a nontrivial minimiser $\psi$ for the variational formula (1.7), we can choose $\eta>0$ so small that the variational formula on the right-hand side of (1.13) is equal to $\Theta(\phi)$. Hence, $\Theta(\phi)>1$, and by Theorem 1.1 we infer that

$$
\mathbb{E}_{x}\left[\exp \left(\sum_{i=1}^{n}\left\langle\phi_{i}^{2 p}, \ell\right\rangle^{1 / p}\right)\right]<\infty
$$

for all $x \in B[\varepsilon]^{p}$ and the intersection local time $\ell$ of the Brownian motion killed upon leaving $B[\varepsilon]$. The desired result for the original domain follows by monotonicity.

Now assume that $l_{\phi, B}^{p}>0$. Then there is a $\psi \in \mathcal{D}(B)$ satisfying $\|\psi\|_{2 p}=1$ and

$$
\frac{p}{2}\|\nabla \psi\|_{2}^{2}<\sum_{i=1}^{n}\left\|\phi_{i} \psi\right\|_{2 p}^{2} .
$$

There exists a domain $U$ with $\bar{U} \subset B$ such that

$$
\frac{p}{2}\|\nabla \psi\|_{2}^{2}<\sum_{i=1}^{n}\left\|\phi_{i} 1_{U} \psi\right\|_{2 p}^{2} .
$$


Multiplying $\psi$ with an appropriate positive constant, we obtain a new $\psi \in \mathcal{D}(B)$ satisfying $\sum_{i=1}^{n}\left\|\phi_{i} 1_{U} \psi\right\|_{2 p}^{2}=1$ and $\frac{p}{2}\|\nabla \psi\|_{2}^{2}<1$, which implies that $\Theta\left(\phi 1_{U}\right)<1$. The result follows from Theorem 1.1 and monotonicity.

Remark 1.4 (Comparison with the result of Pinsky). Looking at the special case $p=1$, and (without loss of generality) $n=1$, Pinsky [Pi86] shows (1.11) for any continuous $\phi_{1}: \bar{B} \rightarrow \mathbb{R}$ in the case that $B$ has a $C^{2}$-boundary. His result is based on the formula

$$
\lim _{t \uparrow \infty} \frac{1}{t} \log \mathbb{E}_{x}\left[\exp \left\{\int_{0}^{t} \phi_{1}(W(s)) d s\right\}, T^{1}>t\right]=l_{\phi_{1}, B}^{1}, \text { for all } x \in B,
$$

which follows from the Donsker-Varadhan theory and an analysis of the regularity of the variational problem defining $l_{\phi, B}^{1}$. Note that our approach requires weaker regularity assumptions, but is not suitable to deal with functions of changing sign. Of course, the main point of our investigation is the generalisation to the case $p>1$, where (1.14) is not available.

In our proof of Theorem 1.1 it is essential to show the existence of minimisers in (1.7) and characterise them by differential equations.

Proposition 1.5 (Analysis of $\Theta(\phi))$. Let $\phi=\left(\phi_{1}, \ldots, \phi_{n}\right)$ be a family of nonnegative bounded measurable functions with compact support in $B$. Then the infimum in (1.7) is attained. Every minimiser $\psi \in \mathcal{D}(B)$ satisfies the equation

$$
-\frac{p}{2} \Delta \psi=\Theta(\phi) \psi^{2 p-1} \sum_{i=1}^{n}\left\|\phi_{i} \psi\right\|_{2 p}^{2-2 p} \phi_{i}^{2 p} .
$$

We do not know in general whether the solution of (1.15) is unique, even in the simpler case $n=1$, where the equation simplifies to the nonlinear eigenvalue equation

$$
-\frac{p}{2} \Delta \psi=\Theta(\phi) \psi^{2 p-1} \phi^{2 p} .
$$

A natural question in this context is whether the minimisers in the variational problem (1.7) allow a probabilistic interpretation. Our second main result provides an interpretation as the asymptotic "shape" of the intersection local time when the total mass in a given set is large.

For the formulation of this result let $U$ be an open, bounded Lebesgue continuity set whose closure is contained in $B$, and define a (random) probability measure $L$ on $U$ as the normalized intersection local times $L=\ell / \ell(U)$ on $U$. Let d denote a metric on the space $\mathcal{M}_{1}(U)$ of probability measures on $U$ that induces the weak topology. Under the conditional law $\mathbb{P}\{\cdot \mid \ell(U)>a\}$, as $a \uparrow \infty$, the measure $L$ satisfies the following law of large numbers.

Theorem 1.6 (Law of Large Masses). Denote by $\mathfrak{M} \subset \mathcal{M}_{1}(U)$ the set of measures $\psi^{2 p}(x) d x$ on $U$ with $\psi$ a minimiser in the variational formula for $\Theta\left(1_{U}\right)$. Then, for any $x \in B^{p}$,

$$
\lim _{a \uparrow \infty} \mathbb{P}_{x}\{\mathrm{~d}(L, \mathfrak{M}) \geq \varepsilon \mid \ell(U)>a\}=0, \quad \text { for any } \varepsilon>0 .
$$

The convergence is exponential with speed $a^{1 / p}$. 
This result was announced, without proof, in KM02. By Proposition 1.5, the densities $\psi^{2 p}$ of the measures in $\mathfrak{M}$ satisfy the nonlinear eigenvalue equation

$$
-\frac{p}{2} \Delta \psi=\Theta\left(1_{U}\right) \psi^{2 p-1} 1_{U}
$$

Whether the solution to this equation is unique, and also whether $\mathfrak{M}$ is a singleton, seems to be an open problem. It is a further open problem to determine the precise rate of convergence in Theorem 1.6.

Remark 1.7 (Comparison with a result of Chen). In a recent paper Chen Ch04] looks at the intersection local time $\ell_{1}$ for $p>1$ Brownian paths in $\mathbb{R}^{d}$ each running up to time one. He finds a sharp criterion for finiteness of the total intersection local time. For $q=\frac{d}{2}(p-1)$ and

$$
\gamma(p, d)=q\left(\frac{p}{p-q}\right)^{1-\frac{p}{q}} \sup \left\{\|\psi\|_{2 p}^{2}-\frac{1}{2}\|\nabla \psi\|_{2}^{2}: \psi \in \mathcal{D}\left(\mathbb{R}^{d}\right),\|\psi\|_{2}=1\right\}^{1-\frac{p}{q}},
$$

he shows that

$$
\mathbb{E}_{x}\left[\exp \left(\gamma \ell_{1}\left(\mathbb{R}^{d}\right)^{2 / d(p-1)}\right)\right]\left\{\begin{array}{l}
<\infty \text { for all } x \in B^{p} \text { if } \gamma<\gamma(p, d), \\
=\infty \text { for all } x \in B^{p} \text { if } \gamma>\gamma(p, d) .
\end{array}\right.
$$

Because of the fixed time horizon this problem is very different from the one we are looking at - note also the completely different scaling behaviour. Still it is interesting to compare the techniques of proof. Chen's method, again quite different from ours, is based on asymptotics for the Feynman-Kac formula for occupation times and approximation of intersection local time by occupation time. Understanding the relationship between these results and methods would probably lead to significant progress in the research programme set out in the introduction.

\section{OVERVIEW AND SETUP OF THE PROOF}

Our main results follow from an analysis of the large- $k$ asymptotics of the $k$ th moments of the random variables

$$
\sum_{i=1}^{n}\left\langle\phi_{i}^{2 p}, \ell\right\rangle^{1 / p}
$$

In Section 2.1 we fix some notation about entropy and relative entropy. In Section 2.2 we derive the moment asymptotics in terms of a variational formula involving relative entropies. We also identify this formula in terms of $\Theta(\phi)$ defined in (1.7). In Section 2.3 we complete the proofs.

2.1. Entropy and relative entropy. For any probability measure $\mu$ and any measure $\widetilde{\mu}$ on the same measure space $X$ the relative entropy or Kullback-Leibler distance of $\mu$ with respect to $\widetilde{\mu}$ is defined as

$$
H(\mu \mid \widetilde{\mu})=\int \mu(d x) \log \frac{\mu(d x)}{\widetilde{\mu}(d x)},
$$

which is to be interpreted as infinity if $\mu \nless \widetilde{\mu}$. If $\widetilde{\mu}=f d x$, then we often write $H(\mu \mid f)$ instead of $H(\mu \mid \widetilde{\mu})$. By Jensen's inequality we always have $H(\mu \mid \widetilde{\mu}) \geq$ $-\log \widetilde{\mu}(X)$ and equality holds if and only if $\mu=\widetilde{\mu} / \widetilde{\mu}(X)$. More specifically, for any $\mu \in \mathcal{M}_{1}(B)$ we define $I(\mu)=H(\mu \mid$ Leb $)$, the relative entropy of $\mu$ with respect to the Lebesgue measure Leb on $B$. For $\lambda \in \mathbb{S}_{n}$, where $\mathbb{S}_{n}$ denotes the $n$-dimensional unit simplex, we write $I(\lambda)=\sum_{i=1}^{n} \lambda_{i} \log \lambda_{i}$ for the relative entropy of $\lambda$ with 
respect to the counting measure. In both cases the functional $I$ is convex and lower semicontinuous. We denote by

$$
\mathcal{M}_{1}^{*}(B)=\left\{\nu \in \mathcal{M}_{1}\left(B^{2}\right): \nu(A \times B)=\nu(B \times A) \text { for all Borel sets } A \subset B\right\}
$$

the set of probability measures $\nu$ on $B^{2}$ with equal marginals $\nu_{1}(A)=\nu(A \times B)$ and $\nu_{2}(A)=\nu(B \times A)$, and we denote the marginal by $\bar{\nu}=\nu_{1}=\nu_{2}$. For $\nu \in \mathcal{M}_{1}\left(B^{2}\right)$ we define

$$
I_{\mu}^{2}(\nu)= \begin{cases}H\left(\nu \mid \nu_{1} \otimes \mu\right), & \text { if } \nu \in \mathcal{M}_{1}^{*}(B) \\ \infty, & \text { otherwise }\end{cases}
$$

It is known that $I_{\mu}^{2}$ is lower semicontinuous and convex.

By $G=G_{B}: \mathbb{R}^{d} \times \mathbb{R}^{d} \rightarrow[0, \infty]$ we denote the Green function of a Brownian motion stopped when reaching $\partial B$. That is,

$$
G(x, y)=\int_{0}^{\infty} p_{s}(x, y) d s, \text { for } x, y \in B
$$

where $p_{s}(x, y)$ denotes the transition sub-probability density of the stopped motion. Define a function $\mathcal{G}: \mathcal{M}_{1}(B) \longrightarrow \mathbb{R}$ by

$$
\begin{aligned}
\mathcal{G}(\mu) & =\inf _{\substack{\nu \in \mathcal{M}_{1}^{*}(B) \\
\bar{\nu}=\mu}}\left\{I_{\mu}^{2}(\nu)-\langle\nu, \log G\rangle\right\} \\
& =\inf _{\substack{\nu \in \mathcal{M} \mathcal{M}_{1}^{*}(B) \\
\bar{\nu}=\mu}} \int_{B} \int_{B} \nu(d x d y) \log \frac{\nu(d x d y)}{\mu(d x) \mu(d y) G(x, y)} .
\end{aligned}
$$

Observe that it suffices to take the infimum over measures $\nu$ satisfying $\nu \ll \mu \otimes \mu$. We can replace $I_{\mu}^{2}(\nu)$ in the definition of $\mathcal{G}$ by either the relative entropy $H(\nu \mid \mu \otimes \mu)$ or the mutual information $H\left(\nu \mid \nu_{1} \otimes \nu_{2}\right)$.

2.2. Moment asymptotics. Let $\phi=\left(\phi_{1}, \ldots, \phi_{n}\right)$ be a family of bounded nonnegative Borel measurable functions with compact support in $B$ and define, for any $\lambda \in \mathbb{S}_{n}$,

$$
\mathfrak{H}(\phi, \lambda):=-\inf \left\{\sum_{i=1}^{n} \lambda_{i} H\left(\mu_{i} \mid \phi_{i}^{2 p}\right)+p \mathcal{G}\left(\sum_{j=1}^{n} \lambda_{j} \mu_{j}\right) \mid \mu_{1}, \ldots, \mu_{n} \in \mathcal{M}_{1}(B)\right\} .
$$

Note that it suffices to take the infimum over measures $\mu_{i}$ that satisfy $\mu_{i} \ll \phi_{i}^{2 p} d x$. The following proposition is the main result of Section 3 ,

Proposition 2.1 (Asymptotics for mixed moments). Fix $x \in B^{p}$.

(i) For every $\lambda \in \mathbb{S}_{n}$,

$$
\liminf _{k \uparrow \infty} \frac{1}{k} \log \mathbb{E}_{x}\left[\frac{1}{k !^{p}} \prod_{i=1}^{n}\left\langle\phi_{i}^{2 p}, \ell\right\rangle^{k \lambda_{i}}\right] \geq \mathfrak{H}(\phi, \lambda) .
$$

(ii) If each $\phi_{i}$ is bounded away from zero on its support, then

$$
\limsup _{k \uparrow \infty} \sup _{\lambda \in \mathbb{S}_{n}}\left[\frac{1}{k} \log \mathbb{E}_{x}\left[\frac{1}{k !^{p}} \prod_{i=1}^{n}\left\langle\phi_{i}^{2 p}, \ell\right\rangle^{k \lambda_{i}}\right]-\mathfrak{H}(\phi, \lambda)\right] \leq 0 .
$$


(iii) Let $U \subset B$ be a bounded open Lebesgue continuity set whose closure is contained in $B$. Let $H \subset \mathcal{M}_{1}(U)$ and $\varepsilon>0$. Then

$$
\limsup _{k \uparrow \infty} \frac{1}{k} \log \mathbb{E}_{x}\left[\frac{1}{k !^{p}} \ell(U)^{k} 1_{\{L \in H\}}\right] \leq-\inf _{\mu \in H[\varepsilon]}\{I(\mu)+p \mathcal{G}(\mu)\},
$$

where $H[\varepsilon] \subset \mathcal{M}_{1}(U)$ denotes the $\varepsilon$-neighbourhood of the set $H$.

In this section we show how to use this result, together with an analysis of the variational problems to complete the proofs of our theorems. We first derive from Proposition 2.1 the moment asymptotics of the random variable $\sum_{i=1}^{n}\left\langle\phi_{i}^{2 p}, \ell\right\rangle^{1 / p}$. For this purpose define

$$
W(\phi)=-\inf _{\lambda \in \mathbb{S}_{n}}\{p I(\lambda)-\mathfrak{H}(\phi, \lambda)\} .
$$

Proposition 2.2. Fix $x \in B^{p}$.

(1) We have

$$
\liminf _{k \uparrow \infty} \frac{1}{k} \log \mathbb{E}_{x}\left[\frac{1}{k ! p}\left(\sum_{i=1}^{n}\left\langle\phi_{i}^{2 p}, \ell\right\rangle^{1 / p}\right)^{k p}\right] \geq W(\phi) .
$$

(2) Assume that every $\phi_{i}$ is bounded away from zero on its support. Then

$$
\limsup _{k \uparrow \infty} \frac{1}{k} \log \mathbb{E}_{x}\left[\frac{1}{k !^{p}}\left(\sum_{i=1}^{n}\left\langle\phi_{i}^{2 p}, \ell\right\rangle^{1 / p}\right)^{k p}\right] \leq W(\phi) .
$$

Proof. We use Proposition 2.1 and denote $\varphi_{i}=\phi_{i}^{2 p}$. For $l, n \in \mathbb{N}$, let

$$
\mathbb{S}_{n}(l)=\left\{\lambda \in \mathbb{S}_{n}: l \lambda_{i} \text { is an integer for all } i\right\} .
$$

The multinomial theorem yields that

$$
\left(\sum_{i=1}^{n}\left\langle\varphi_{i}, \ell\right\rangle^{1 / p}\right)^{k p}=\sum_{\lambda \in \mathbb{S}_{n}(k p)}\left(\begin{array}{c}
k p \\
k p \lambda_{1}, \ldots, k p \lambda_{n}
\end{array}\right) \prod_{i=1}^{n}\left\langle\varphi_{i}, \ell\right\rangle^{k \lambda_{i}} .
$$

Stirling's formula yields that, uniformly in $\lambda \in \mathbb{S}_{n}(k p)$, as $k \uparrow \infty$,

$$
\left(\begin{array}{c}
k p \\
k p \lambda_{1}, \ldots, k p \lambda_{n}
\end{array}\right)=e^{-k p I(\lambda)} e^{o(k)} .
$$

To prove part (i) of Proposition 2.2, pick some small $\eta>0$ and choose an approximate minimiser $\lambda^{*} \in \mathbb{S}_{n}$ in (2.9) such that

$$
p I\left(\lambda^{*}\right)-\mathfrak{H}\left(\phi, \lambda^{*}\right) \leq-W(\phi)+\eta \text {. }
$$

Pick $\lambda \in \mathbb{S}_{n}(k p)$ such that $\left|\lambda_{i}-\lambda_{i}^{*}\right| \leq \frac{1}{k}$ for any $i \in\{1, \ldots, n\}$. Note that the vector $\widetilde{\lambda}=\frac{1}{\varepsilon}\left(\lambda^{*}-\lambda(1-\varepsilon)\right)$ lies in $\mathbb{S}_{n}$. Fix $\varepsilon>0$ and use Hölder's inequality to derive

$$
\mathbb{E}_{x}\left[\prod_{i=1}^{n}\left\langle\varphi_{i}, \ell\right\rangle^{k \lambda_{i}}\right] \geq \mathbb{E}_{x}\left[\prod_{i=1}^{n}\left\langle\varphi_{i}, \ell\right\rangle^{k \lambda_{i}^{*}}\right]^{\frac{1}{1-\varepsilon}} \mathbb{E}_{x}\left[\prod_{i=1}^{n}\left\langle\varphi_{i}, \ell\right\rangle^{k \tilde{\lambda}_{i}}\right]^{-\frac{\varepsilon}{1-\varepsilon}}
$$

The last term is further estimated using Hölder's inequality by

$$
\mathbb{E}_{x}\left[\prod_{i=1}^{n}\left\langle\varphi_{i}, \ell\right\rangle^{k \tilde{\lambda}_{i}}\right]^{-\frac{\varepsilon}{1-\varepsilon}} \geq \prod_{i=1}^{n} \mathbb{E}_{x}\left[\left\langle\varphi_{i}, \ell\right\rangle^{k}\right]^{-\varepsilon \tilde{\lambda}_{i} /(1-\varepsilon)} \geq\left(k !^{p} e^{C k}\right)^{-\frac{\varepsilon}{1-\varepsilon}}
$$

where $C$ only depends on $p$ and $\varphi_{1}, \ldots, \varphi_{n}$. This follows from rough a priori estimates based on Le Gall's moment formula; see e.g. [LG87, Proposition 3]. By 
continuity and nonpositivity of $I$, we may estimate $I(\lambda) \leq I\left(\lambda^{*}\right) \frac{1-2 \varepsilon}{1-\varepsilon}$, for $k$ sufficiently large. We take expectations on both sides of (2.12), and obtain a lower bound by restricting the sum on the right side to the unique summand for $\lambda$ chosen above, obtaining, with the help of Proposition 2.1(i),

$$
\begin{aligned}
\liminf _{k \uparrow \infty} & \frac{1}{k} \log \mathbb{E}_{x}\left[\frac{1}{k ! p}\left(\sum_{i=1}^{n}\left\langle\phi_{i}^{2 p}, \ell\right\rangle^{1 / p}\right)^{k p}\right] \\
& \geq-p I(\lambda)+\frac{1}{1-\varepsilon} \liminf _{k \uparrow \infty} \frac{1}{k} \log \mathbb{E}_{x}\left[\frac{1}{k ! p} \prod_{i=1}^{n}\left\langle\phi_{i}^{2 p}, \ell\right\rangle^{k \lambda_{i}^{*}}\right]-\frac{\varepsilon C}{1-\varepsilon} \\
& \geq-\frac{1}{1-\varepsilon}\left[p I\left(\lambda^{*}\right)-\mathfrak{H}\left(\phi, \lambda^{*}\right)+\varepsilon C-2 p \varepsilon I\left(\lambda^{*}\right)\right] \\
& \geq-\frac{1}{1-\varepsilon}\left[-W(\phi)+\eta+\varepsilon C-2 p \varepsilon I\left(\lambda^{*}\right)\right] .
\end{aligned}
$$

Now let $\eta \downarrow 0$ and $\varepsilon \downarrow 0$ to arrive at (2.10).

Now we prove Proposition 2.2(ii). For any small $\delta>0$ we have, by Proposition 2.1(ii), for sufficiently large $k$ and any $\lambda \in \mathbb{S}_{n}$,

$$
\frac{1}{k} \log \mathbb{E}_{x}\left[\frac{1}{k ! p} \prod_{i=1}^{n}\left\langle\varphi_{i}, \ell\right\rangle^{k \lambda_{i}}\right] \leq \mathfrak{H}(\phi, \lambda)+\delta .
$$

Hence, by (2.12), (2.13), and using that the cardinality of $\mathbb{S}_{n}(k p)$ is bounded by $(k p)^{n}=e^{o(k)}$, we get

$$
\limsup _{k \uparrow \infty} \frac{1}{k} \log \mathbb{E}_{x}\left[\frac{1}{k ! p}\left(\sum_{i=1}^{n}\left\langle\phi_{i}^{2 p}, \ell\right\rangle^{1 / p}\right)^{k p}\right] \leq-\inf _{\lambda \in \mathbb{S}_{n}}[p I(\lambda)-\mathfrak{H}(\phi, \lambda)]+\delta .
$$

Now we let $\delta \downarrow 0$ in (2.16) to complete the proof.

At this point we would like to replace the cumbersome expression (2.9) for $W(\phi)$ with a more elegant expression involving energies of functions. The following result is the main result of Section 4.

Proposition 2.3. Let $\Theta(\phi)$ be as in (1.7). Then

$$
W(\phi)=-p \log \frac{\Theta(\phi)}{p} .
$$

Moreover, in the variational problems (1.7) and (2.9) (substituting (2.6) ) minimisers exist, and they are related by the formulas

$$
\lambda_{i}=\left\|\psi \phi_{i}\right\|_{2 p}^{2} \quad \text { and } \quad \psi^{2 p} \phi_{i}^{2 p}=\lambda_{i}^{p} \frac{d \mu_{i}}{d x}, \quad \text { for } i=1, \ldots, n .
$$

Using this, we arrive at the following theorem.

Theorem 2.4 (Moment asymptotics). Let $\phi=\left(\phi_{1}, \ldots, \phi_{n}\right)$ be a family of bounded nonnegative Borel measurable functions with compact support in $B$, and let $\Theta(\phi)$ be as in (1.7). Then, for all $x \in B^{p}$,

$$
\lim _{k \uparrow \infty} \frac{1}{k} \log \mathbb{E}_{x}\left[\frac{1}{k !^{p}}\left(\sum_{i=1}^{n}\left\langle\phi_{i}^{2 p}, \ell\right\rangle^{1 / p}\right)^{k p}\right]=-p \log \frac{\Theta(\phi)}{p} .
$$


Proof. By Propositions 2.2 and 2.3 we have the result if $\phi_{1}, \ldots, \phi_{n}$ are bounded away from zero on their respective supports. In order to remove this restriction, let $\phi_{1}, \ldots, \phi_{n}$ be as in the statement and define $\phi_{i}^{(\varepsilon)}=\phi_{i}+\varepsilon 1_{\operatorname{supp}\left(\phi_{i}\right)}$ for $\varepsilon>0$. All we have to show is

$$
\limsup _{\varepsilon \downarrow 0} \Theta\left(\phi^{(\varepsilon)}\right) \geq \Theta(\phi) .
$$

It is clear that the condition in the variational problem (1.7) may be relaxed to

$$
\Theta(\phi)=\inf \left\{\frac{p}{2}\|\nabla \psi\|_{2}^{2}: \psi \in \mathcal{D}(B), \sum_{i=1}^{n}\left\|\phi_{i} \psi\right\|_{2 p}^{2} \geq 1\right\} .
$$

Now we argue that there is $M>0$ such that, for any $\varepsilon \in[0,1]$,

$$
\Theta\left(\phi^{(\varepsilon)}\right)=\inf \left\{\frac{p}{2}\|\nabla \psi\|_{2}^{2}: \psi \in \mathcal{D}(B), \sum_{i=1}^{n}\left\|\phi_{i}^{(\varepsilon)} \psi\right\|_{2 p}^{2} \geq 1,\left\|1_{U} \psi\right\|_{2 p} \leq M\right\},
$$

where $U$ denotes the union of the supports of $\phi_{1}, \ldots, \phi_{n}$. Indeed, note that $\varepsilon \mapsto$ $\Theta\left(\phi^{(\varepsilon)}\right)$ is decreasing and therefore bounded on $[0,1]$. Hence, in dimensions $d \geq 3$, (2.19) follows from (5.2), and in $d \leq 2$ it follows from (5.3).

For any $\varepsilon \in[0,1]$ and any $\psi$ in the set on the right-hand side of (2.19), we have

$$
\left\|\phi_{i}^{(\varepsilon)} \psi\right\|_{2 p}^{2} \leq\left\|\phi_{i} \psi\right\|_{2 p}^{2}+\varepsilon C,
$$

where $C>0$ only depends on $M$ and on the suprema of $\phi_{1}, \ldots, \phi_{n}$. Now choose $\varepsilon \in[0,1 /(2 C)]$. Then we have

$$
\begin{aligned}
\Theta\left(\phi^{(\varepsilon)}\right) & \geq \inf \left\{\frac{p}{2}\|\nabla \psi\|_{2}^{2}: \psi \in \mathcal{D}(B), \sum_{i=1}^{n}\left\|\phi_{i} \psi\right\|_{2 p}^{2} \geq 1-\varepsilon C,\left\|1_{U} \psi\right\|_{2 p} \leq M\right\} \\
& \geq \inf \left\{\frac{p}{2}\|\nabla \psi\|_{2}^{2}: \psi \in \mathcal{D}(B), \sum_{i=1}^{n}\left\|\phi_{i} \psi\right\|_{2 p}^{2} \geq 1-\varepsilon C\right\}=\frac{\Theta(\phi)}{1-\varepsilon C},
\end{aligned}
$$

which completes the proof.

2.3. Completion of the proofs. With this result at hand we can easily complete the proof of our main results. The relation between large moment asymptotics and exponential moments is given by the following easy lemma.

Lemma 2.5. Fix $p>0$ and let $X$ be a nonnegative random variable such that

$$
\lim _{k \uparrow \infty} \frac{1}{k} \log E\left[\frac{1}{k !^{p}} X^{k p}\right]=-p \log \frac{\Theta}{p}
$$

exists for some $\Theta>0$. Then we have $E\left[e^{X}\right]<\infty$ if $\Theta>1$, and $E\left[e^{X}\right]=\infty$ if $\Theta<1$.

Proof. The assumption and Stirling's formula imply that, as $k \uparrow \infty$,

$$
E\left[X^{k p}\right]=k !^{p}\left(\frac{p}{\Theta}\right)^{k p} e^{o(k)}=(k p) ! \Theta^{-p k} e^{o(k)} .
$$

In the case $\Theta<1$, this shows that the terms $E\left[X^{m}\right] / m$ ! are not summable over the subsequence $m=k p, k \in \mathbb{N}$, which implies the second statement. In the case $\Theta>1$ we estimate, for any $m$ such that $k p \leq m \leq(k+1) p$, with the help of Hölder's 
inequality and Stirling's formula,

$E\left[X^{m}\right] \leq E\left[X^{k p}\right]^{m /(k p)}=k !^{m / k}\left(\frac{p}{\Theta}\right)^{m} e^{o(m)} \leq\left(\frac{m}{p e}\right)^{m}\left(\frac{p}{\Theta}\right)^{m} e^{o(m)}=m ! \Theta^{-m} e^{o(m)}$.

The first statement follows by summing over all $n$.

Proof of Theorem 1.1. Apply Lemma 2.5 to the situation of Theorem 2.4 to get (1.8). Similarly to Lemma 2.5, KM02, Lemma 2.3] relates large integer moments and upper tail asymptotics, and allows us to infer (1.9) from Theorem 2.4 .

Proof of Theorem 1.6. Suppose $H \subset \mathcal{M}_{1}(U)$ is such that $H[\varepsilon]$, an $\varepsilon$-neighbourhood of $H$, is disjoint from $\mathfrak{M}$. By (2.17), in the special case $n=1, \phi_{1}=1_{U}$, the set $\mathfrak{M}$ is equal to the set of minimisers of $I+p \mathcal{G}$ over the set $\mathcal{M}_{1}(U)$. As the set of minimisers is closed, we can find $\varepsilon>0$ such that $I(\mu)+p \mathcal{G}(\mu)-\inf \{I+p \mathcal{G}\}>\varepsilon$ for all $\mu \in H[\varepsilon]$. Now,

$$
\begin{aligned}
\frac{1}{a^{1 / p}} \log \mathbb{P}\{L & \in H \mid \ell(U)>a\} \\
& =\frac{1}{a^{1 / p}} \log \mathbb{P}\{\ell(U)>a \mid L \in H\}-\frac{1}{a^{1 / p}} \log \mathbb{P}\{\ell(U)>a\} .
\end{aligned}
$$

The second term converges to $-p \exp \left(\frac{1}{p} \inf \{I+p \mathcal{G}\}\right)$ by KM02, Theorem 1.1 and Proposition 2.1]. For the first term we use the Tauberian Theorem KM02, Lemma 2.3], together with Proposition 2.1(iii) to obtain

$$
\begin{aligned}
& \lim _{a \uparrow \infty} \frac{1}{a^{1 / p}} \log \mathbb{P}\{\ell(U)>a \mid L \in H\} \\
& \quad \leq-p \exp \left(-\frac{1}{p} \lim _{k \uparrow \infty} \frac{1}{k} \log \mathbb{E}_{x}\left[\frac{1}{k ! p} \ell(U)^{k} \mid L \in H\right]\right) \\
& \quad=-p \exp \left(-\frac{1}{p} \lim _{k \uparrow \infty} \frac{1}{k} \log \mathbb{E}_{x}\left[\frac{1}{k !^{p}} \ell(U)^{k} 1_{\{L \in H\}}\right]\right) \\
& \quad \leq-p \exp \left(\frac{1}{p} \inf _{\mu \in H[\varepsilon]}\{I(\mu)+p \mathcal{G}(\mu)\}\right) .
\end{aligned}
$$

Altogether,

$$
\begin{aligned}
\limsup _{a \uparrow \infty} & \frac{1}{a^{1 / p}} \log \mathbb{P}\{L \in H \mid \ell(U)>a\} \\
& \leq-p \exp \left(\frac{1}{p} \inf _{\mu \in H[\varepsilon]}\{I(\mu)+p \mathcal{G}(\mu)\}\right)+p \exp \left(\frac{1}{p} \inf \{I+p \mathcal{G}\}\right)<0,
\end{aligned}
$$

which implies the result.

\section{Moment Asymptotics}

In this section we prove Proposition 2.1. The proof is an extension of the proof of [KM02, Theorem 1.1], and we are able to use some material from there. To keep the notation manageable we assume that $x=(0, \ldots, 0)$, i.e., all motions are started in the origin. We write $\mathbb{E}$ instead of $\mathbb{E}_{0}$. The case of arbitrary starting points does not pose any additional difficulties for our asymptotic statements. We define $\varphi_{i}=\phi_{i}^{2 p}$.

The proof is based on a moment formula of Le Gall [LG86]. To formulate the result in the necessary generality, recall the Green function $G=G_{B}$ and define the 
function $\mathbb{P}_{k}: B^{k} \rightarrow \mathbb{R}$ by

$$
\mathbb{P}_{k}(y)=\frac{1}{k !} \sum_{\sigma \in \mathfrak{S}_{k}} \prod_{i=1}^{k} G\left(y_{\sigma(i-1)}, y_{\sigma(i)}\right), \text { for } y=\left(y_{1}, \ldots, y_{k}\right) \in B^{k},
$$

where we put $y_{0}=0$, the starting point of the motions. $\mathfrak{S}_{k}$ is the symmetric group in $k$ elements and we write elements $\sigma \in \mathfrak{S}_{k}$ as permutations $\sigma:\{1, \ldots, k\} \rightarrow$ $\{1, \ldots, k\}$ and agree on the additional convention that $\sigma(0)=0$. Introduce the empirical measure of the vector $y=\left(y_{1}, \ldots, y_{k}\right) \in B^{k}$,

$$
L_{y, k}=\frac{1}{k} \sum_{j=1}^{k} \delta_{y_{j}} \in \mathcal{M}_{1}(B)
$$

and note that $\Phi_{k}$ is a permutation symmetric function, i.e., it depends on $y$ only via $L_{y, k} . \Phi_{k}$ assumes the value $\infty$ if and only if $0, y_{1}, \ldots, y_{k}$ are not pairwise distinct. For the rest of the proof, we tacitly assume that the numbers $k \lambda_{1}, \ldots, k \lambda_{n}$ are integers. This simplification can be justified by simple local approximations of the type $\lambda_{i} \rightsquigarrow\left\lfloor k \lambda_{i}\right\rfloor / k$ or $\lambda_{i} \rightsquigarrow\left\lceil k \lambda_{i}\right\rceil / k$. Inequalities (2.14) and (2.15) show that this does not affect the asymptotics on the left-hand side of (2.7) and (2.8).

In the following, we organise the vector $y$ as

$$
y=\left(y_{j}^{i}: j=1, \ldots, k \lambda_{i}, \text { and } i=1, \ldots, n\right) .
$$

Lemma 3.1 (Moment formulas).

(1) For all integers $k \geq 1$,

$$
\mathbb{E}\left[\frac{1}{k !^{p}} \prod_{i=1}^{n}\left\langle\varphi_{i}, \ell\right\rangle^{k \lambda_{i}}\right]=\int_{B^{k}} d y\left(\Phi_{k}(y)\right)^{p} \prod_{i=1}^{n} \prod_{j=1}^{\lambda_{i} k} \varphi_{i}\left(y_{j}^{i}\right) .
$$

(2) Let $H \subset \mathcal{M}_{1}(U)$ be a Borel set, and $\delta>0$. Then, for all sufficiently large $k \in \mathbb{N}$

$$
\mathbb{E}\left[\frac{1}{k !^{p}} \ell(U)^{k} 1_{\{L \in H\}}\right] \leq 2 \int_{U^{k}} d y 1_{\left\{L_{y, k} \in H[\delta]\right\}}\left(\Phi_{k}(y)\right)^{p},
$$

where $H[\delta]$ is the $\delta$-neighbourhood of $H$.

Proof. A variant of part (i) was proved in [LG86; see [LG87, (2c)]. We focus on part (ii), where the main idea is that the random variable $L$ is asymptotically estimated in terms of the empirical measure $L_{y, k}$ induced by the integration variable $y=\left(y_{1}, \ldots, y_{k}\right)$ on the right-hand side of the moment formula.

We now suppose $\delta>0$ and a Borel set $H \subset \mathcal{M}_{1}\left(\mathbb{R}^{d}\right)$ are given. Then

$$
\begin{aligned}
& \mathbb{E}\left[\ell(U)^{k} 1_{\{L \in H\}}\right] \\
& =\mathbb{E}\left[\int_{U^{k}} \ell^{\otimes k}(d y) 1_{\left\{L_{y, k} \in H[\delta / 2]\right\}}\right]+\mathbb{E}\left[\ell(U)^{k} \mathrm{E}_{L^{\otimes k}}\left[1_{\{L \in H\}}-1_{\left\{L_{Y, k} \in H[\delta / 2]\right\}}\right]\right] \\
& \leq \mathbb{E}\left[\int_{U^{k}} \ell^{\otimes k}(d y) 1_{\left\{L_{y, k} \in H[\delta / 2]\right\}}\right]+\mathbb{E}\left[\ell(U)^{k} 1_{\{L \in H\}} L^{\otimes k}\left\{d\left(L, L_{Y, k}\right) \geq \delta / 3\right\}\right],
\end{aligned}
$$

where $\mathrm{E}_{L \otimes k}$ denotes expectation with respect to $L^{\otimes k}$, and $Y: U^{k} \rightarrow U^{k}$ is the identity map, i.e., $Y_{1}, \ldots, Y_{k}$ are independent with distribution $L$. 
We now look at the first term on the right-hand side of (3.4) and show that

$$
\begin{aligned}
& \mathbb{E}\left[\int_{U^{k}} \ell^{\otimes k}(d y) 1_{\left\{L_{y, k} \in H[\delta / 2]\right\}}\right] \\
& \quad \leq \int_{U^{k}} d y 1_{\left\{L_{y, k} \in H[\delta]\right\}}\left(\sum_{\sigma \in \mathfrak{S}_{k}} \prod_{i=1}^{k} G\left(y_{\sigma(i-1)}, y_{\sigma(i)}\right)\right)^{p} .
\end{aligned}
$$

This requires us to distinguish between the dimensions. In $d=1$ we have that

$$
\mathbb{E}\left[\int_{U^{k}} \ell^{\otimes k}(d y) 1_{\left\{L_{y, k} \in H[\delta / 2]\right\}}\right]=\int_{U^{k}} d y 1_{\left\{L_{y, k} \in H[\delta / 2]\right\}}\left(\mathbb{E}\left[\prod_{j=1}^{k} L\left(y_{j}\right)\right]\right)^{p},
$$

where $L(x), x \in U$, is the local time of the stopped process in $x$. Using the approximation of $L(x)$ by the occupation time of $(x, x+\varepsilon)$ it is easily seen that

$$
\mathbb{E}\left[\prod_{j=1}^{k} L\left(y_{j}\right)\right]=\sum_{\sigma \in \mathfrak{S}_{k}} \prod_{i=1}^{k} G\left(y_{\sigma(i-1)}, y_{\sigma(i)}\right)
$$

which proves (3.5) in the case $d=1$.

If $d \geq 2$, an essential ingredient of the proof is Le Gall's Wiener sausage characterization of $\ell$; see [LG86, Th. 3.1]. For every $\varepsilon>0$ define the Wiener sausage around $W_{i}$ by

$$
S_{\varepsilon}^{i}=\left\{x \in B: \text { there is } t \in\left[0, T_{i}\right) \text { with }\left|x-W_{i}(t)\right|<\varepsilon\right\} \text {, for } i=1, \ldots, p,
$$

and their intersection $S_{\varepsilon}=\bigcap_{i=1}^{p} S_{\varepsilon}^{i}$. Recall that $S=W^{1}\left[0, T^{1}\right] \cap \ldots \cap W^{p}\left[0, T^{p}\right]$ and observe that $S=\bigcap_{\varepsilon>0} S_{\varepsilon}$ is the intersection of the $p$ independent Brownian paths. Define

$$
s_{d}(\varepsilon)= \begin{cases}\pi^{-p} \log ^{p}(1 / \varepsilon), & \text { if } d=2, \\ (2 \pi \varepsilon)^{-2}, & \text { if } d=3 \text { and } p=2, \\ \frac{2}{\omega_{d}(d-2)} \varepsilon^{2-d}, & \text { if } d \geq 3 \text { and } p=1,\end{cases}
$$

where $\omega_{d}$ is the volume of the $d$-dimensional unit ball. For every set $A \subset B$ that is almost surely an $\ell$-continuity set,

$$
\lim _{\varepsilon \downarrow 0} s_{d}(\varepsilon) \operatorname{Leb}\left(S_{\varepsilon} \cap A\right)=\ell(A),
$$

in the $L^{k}(\mathbb{P})$-sense for all positive integers $k$ and, in particular, in probability. As we have assumed that $U$ is a Lebesgue continuity set, we see from the moment formula (3.2) for $n=k=1$ and $\varphi_{1}=1_{\partial U}$ that almost surely $\ell(\partial U)=0$ and thus (3.8) applies to the set $A=U$.

Recall from [LG86] that the family of functions

$$
y \mapsto s_{d}(\varepsilon)^{k} \mathbb{P}\left\{y_{j} \in S_{\varepsilon} \text { for all } j\right\}, \quad \text { for } \varepsilon \in\left(0, \frac{1}{2}\right),
$$

is dominated by an integrable function, and

$$
\lim _{\varepsilon \downarrow 0} s_{d}(\varepsilon)^{k} \mathbb{P}\left\{y_{j} \in S_{\varepsilon} \text { for all } j\right\}=\left(\sum_{\sigma \in \mathfrak{S}_{k}} \prod_{i=1}^{k} G\left(y_{\sigma(i-1)}, y_{\sigma(i)}\right)\right)^{p} .
$$


Hence for the first term on the right-hand side of (3.4), by (3.8), Fubini's Theorem and the theorem of dominated convergence,

$$
\begin{aligned}
\mathbb{E}\left[\int_{U^{k}} \ell^{\otimes k}(d y)\right. & \left.1_{\left\{L_{y, k} \in H[\delta / 2]\right\}}\right] \leq \lim _{\varepsilon \downarrow 0} s_{d}(\varepsilon)^{k} \mathbb{E}\left[\int_{\left(U \cap S_{\varepsilon}\right)^{k}} d y 1_{\left\{L_{y, k} \in H[\delta]\right\}}\right] \\
= & \lim _{\varepsilon \downarrow 0} \int_{U^{k}} d y 1_{\left\{L_{y, k} \in H[\delta]\right\}} s_{d}(\varepsilon)^{k} \mathbb{P}\left\{y_{j} \in S_{\varepsilon} \text { for all } j\right\} \\
& =\int_{U^{k}} d y 1_{\left\{L_{y, k} \in H[\delta]\right\}} \lim _{\varepsilon \downarrow 0} s_{d}(\varepsilon)^{k} \mathbb{P}\left\{y_{j} \in S_{\varepsilon} \text { for all } j\right\} \\
& =\int_{U^{k}} d y 1_{\left\{L_{y, k} \in H[\delta]\right\}}\left(\sum_{\sigma \in \mathfrak{S}_{k}} \prod_{i=1}^{k} G\left(y_{\sigma(i-1)}, y_{\sigma(i)}\right)\right)^{p}
\end{aligned}
$$

which is equal to the right-hand side in our claim (3.3).

To derive an upper bound for the second term of the right-hand side of (3.4) that is negligible with respect to the left-hand side in (3.3), we need an upper bound for $L^{\otimes k}\left\{d\left(L, L_{Y, k}\right) \geq \delta / 3\right\}$, which does not depend on the Brownian paths. This will be based on a simple metric-entropy type argument. We first use the fact that the weak topology of measures can be approximated by a finite partition. More precisely, for the given $\delta>0$, we can find a finite partition $\mathcal{P}$ of $B$ and an $\eta=\eta(\delta)>0$ such that,

$$
\sup _{M \in \mathcal{P}}|\nu(M)-\mu(M)| \leq \eta \quad \text { implies } \quad d(\nu, \mu)<\delta / 3 .
$$

Denote the cardinality of $\mathcal{P}$ by $N$ and the natural projection by $\pi: B \rightarrow\{1, \ldots, N\}$ and recall that for any two probability measures $P, Q$ on $\{1, \ldots, N\}$ the KullbackLeibler distance $H(P \mid Q)$ is bounded from below by

$$
\frac{1}{2} \sup _{A \subset\{1, \ldots, N\}}|P(A)-Q(A)|^{2} .
$$

Hence, we obtain for any two probability measures $\nu, \mu$ on $\mathbb{R}^{d}$ that

$$
d(\nu, \mu) \geq \delta / 3 \quad \text { implies } \quad H\left(\nu \circ \pi^{-1} \mid \mu \circ \pi^{-1}\right) \geq \eta^{2} / 2 .
$$

Denote by $\Gamma$ the set of probability measures $Q$ on $\{1, \ldots, N\}$ with $H\left(Q \mid L \circ \pi^{-1}\right) \geq$ $\eta^{2} / 2$. From the upper bound in Sanov's Theorem for the finite alphabet $\{1, \ldots, N\}$ (see [DZ98, p. 15]) we infer that

$$
\begin{aligned}
L^{\otimes k}\left\{d\left(L, L_{Y, k}\right) \geq \delta / 3\right\} & \leq(k+1)^{N} \exp \left(-k \inf _{Q \in \Gamma} H\left(Q \mid L \circ \pi^{-1}\right)\right) \\
& \leq(k+1)^{N} \exp \left(-k \eta^{2} / 2\right),
\end{aligned}
$$

which is the required upper bound. We infer from this that

$$
\begin{aligned}
\mathbb{E}\left[\ell(U)^{k} 1_{\{L \in H\}} L^{\otimes k}\left\{d\left(L, L_{Y, k}\right) \geq \delta / 3\right\}\right] \\
\leq(k+1)^{N} \exp \left(-k \eta^{2} / 2\right) \mathbb{E}\left[\ell(U)^{k} 1_{\{L \in H\}}\right],
\end{aligned}
$$


and putting (3.4), (3.5) and (3.11) together, we obtain

$$
\begin{aligned}
& \left(1-(k+1)^{N} e^{-k \eta^{2} / 2}\right) \mathbb{E}\left[\ell(U)^{k} 1_{\{L \in H\}}\right] \\
& \leq \int_{U^{k}} d y 1_{\left\{L_{y, k} \in H[\delta]\right\}}\left(\sum_{\sigma \in \mathfrak{S}_{k}} \prod_{i=1}^{k} G\left(y_{\sigma(i-1)}, y_{\sigma(i)}\right)\right)^{p} .
\end{aligned}
$$

Finally, $1-(k+1)^{N} \exp \left(-k \eta^{2} / 2\right)>1 / 2$ for all sufficiently large $k$, and this finishes the proof.

In order to derive the upper bounds in Proposition 2.1 it is necessary to replace the Green function $G$ in the definition of $\Phi_{k}(y)$ by some bounded function. We achieve this by cutting off at a large level and show that this does not change the exponential rate of $\Phi_{k}(y)$ asymptotically as the cut-off level gets large. Introduce, for $M \geq 0$, the cut-off Green function $G_{M}=G \wedge M$, and denote

$$
\Phi_{k, M}(y)=\frac{1}{k !} \sum_{\sigma \in \mathfrak{S}_{k}} \prod_{i=1}^{k} G_{M}\left(y_{\sigma(i-1)}, y_{\sigma(i)}\right), \quad \text { for } y \in B^{k} .
$$

The following lemma provides the cutting argument:

Lemma 3.2. There is $C_{0}>0$ and, for all sufficiently large $M>1$ and small $\eta \in(0,1)$, there are constants $C_{M}>0$ and $\varepsilon_{\eta}>0$ such that $\lim _{M \uparrow \infty} C_{M}=$ $\lim _{\eta \downarrow 0} \varepsilon_{\eta}=0$, and the following holds:

(1) For any $k \in \mathbb{N}$ and for any $\lambda \in \mathbb{S}_{n}$,

$$
\begin{aligned}
\int_{B^{k}} d y\left(\Phi_{k}(y)\right)^{p} \prod_{i=1}^{n} \prod_{j=1}^{\lambda_{i} k} \varphi_{i}\left(y_{j}^{i}\right) & \\
\leq 2^{p} p k\left(2 C_{0}\right)^{k} C_{M}^{\eta k} & \\
& +2^{p}\left(1+\varepsilon_{\eta}\right)^{k} p \sum_{m=\lceil k(1-p \eta)\rceil}^{k} \int_{B^{m}} d y\left(\Phi_{m, M}(y)\right)^{p} \prod_{i=1}^{n} \prod_{j=1}^{\lambda_{i} m} \varphi_{i}\left(y_{j}^{i}\right) .
\end{aligned}
$$

(2) For any $H \subset \mathcal{M}_{1}(U)$ Borel, and $\delta>0$, one can pick $\eta>0$ small enough such that

$$
\begin{aligned}
\int_{U^{k}} d y & \left(\Phi_{k}(y)\right)^{p} 1_{\left\{L_{y, k} \in H\right\}} \\
\leq & 2^{p} p k\left(2 C_{0}\right)^{k} C_{M}^{\eta k} \\
& +2^{p}\left(1+\varepsilon_{\eta}\right)^{k} p \sum_{m=\lceil k(1-p \eta)\rceil}^{k} \int_{U^{m}} d y\left(\Phi_{m, M}(y)\right)^{p} 1_{\left\{L_{y, m} \in H[\delta]\right\}}
\end{aligned}
$$

Proof. This follows from an obvious adaptation of Lemmas 3.2 and 3.3 in KM02 and their proofs (recall that $\varphi_{1}, \ldots, \varphi_{n}$ are bounded).

Note that for the upper bounds we may now focus on the $m$-fold integral on the right-hand side of (3.13), resp. (3.14). Observe that the integration domain $B^{m}$ may be replaced by the compact set $U^{m}$, where $U$ is the union of the supports of $\varphi_{i}$ for $i=1, \ldots, n$. 
Our second main technical tool is a reduction to a discrete counting argument. For this purpose, we introduce a finite partition of $U$ which is carefully chosen in order to represent many details of the continuous picture.

To introduce appropriate notation, let $\Sigma_{r}=\{1, \ldots, r\}$ and denote the partition sets by $U_{1}, \ldots, U_{r}$. We assume that every $U_{l}$ is measurable and has positive Lebesgue measure. In Lemma 3.3 below we shall make precise how fine we choose the partition. We call $\pi: U \rightarrow \Sigma_{r}$ the canonical projection, that is, $x \in U_{\pi x}$ for any $x \in U$. We write $\pi y=\left(\left(\pi y_{1}^{i}, \ldots, \pi y_{\lambda_{i} m}^{i}\right): i=1, \ldots, n\right)$ if $y=\left(\left(y_{1}^{i}, \ldots, y_{\lambda_{i} m}^{i}\right): i=1, \ldots, n\right)$. If $\mu$ is a probability measure on $U$, then $\pi \mu \in \mathcal{M}_{1}\left(\Sigma_{r}\right)$ is its projection on $\Sigma_{r}$. Similarly for $\nu \in \mathcal{M}_{1}\left(U^{2}\right)$ we denote the projection on $\Sigma_{r}^{2}$ by $\pi \nu \in \mathcal{M}_{1}\left(\Sigma_{r}^{2}\right)$. If $v$ is in the set $\mathcal{M}_{1}^{*}\left(\Sigma_{r}\right)$ of probability measures on $\Sigma_{r}^{2}$ with equal marginals, we denote by $\bar{v} \in \mathcal{M}_{1}\left(\Sigma_{r}\right)$ its left or right marginal measure. Note that $\pi \bar{\nu}=\overline{\pi \nu}$ for any $\nu \in \mathcal{M}_{1}^{*}(U)$, where $\bar{\nu}$ is the marginal measure of $\nu$.

For measures $u \in \mathcal{M}_{1}\left(\Sigma_{r}\right)$ and $v \in \mathcal{M}_{1}\left(\Sigma_{r}^{2}\right)$ we define discrete analogues of the relative entropy functionals $I$ and $I_{\mu}^{2}$ by

$$
\widetilde{I}(u)=\sum_{l \in \Sigma_{r}} u_{l} \log \frac{u_{l}}{\left|U_{l}\right|} \quad \text { and } \quad \widetilde{I}_{u}^{2}(v)=\sum_{l, m \in \Sigma_{r}} v_{l, m} \log \frac{v_{l, m}}{\bar{v}_{l} u_{m}},
$$

using the usual convention $0 \log 0=0$. Recall that $G_{M}=G \wedge M$ and define the approximate Green functions $G_{M}^{+}, G^{-}: \Sigma_{r}^{2} \rightarrow \mathbb{R}$ by

$$
G_{M}^{+}(l, m)=\sup _{\substack{x \in U_{l} \\ y \in U_{m}}} G_{M}(x, y) \quad \text { and } \quad G^{-}(l, m)=\inf _{\substack{x \in U_{l} \\ y \in U_{m}}} G(x, y) .
$$

Functions $\mathcal{G}_{M}^{+}$and $\mathcal{G}^{-}$on $\mathcal{M}_{1}\left(\Sigma_{r}\right)$ analogous to $\mathcal{G}$ in (2.5) are defined by

$$
\begin{aligned}
& \mathcal{G}_{M}^{+}(u)=\inf _{\substack{v \in \mathcal{M}^{*}\left(\Sigma_{r}\right) \\
v=u}}\left\{\widetilde{I}_{u}^{2}(v)-\left\langle v, \log G_{M}^{+}\right\rangle\right\}, \\
& \mathcal{G}^{-}(u)=\inf _{\substack{v \in \mathcal{M}_{i}^{*}\left(\Sigma_{r}\right) \\
v=u}}\left\{\widetilde{I}_{u}^{2}(v)-\left\langle v, \log G^{-}\right\rangle\right\},
\end{aligned}
$$

where we used the notation $\langle v, F\rangle=\sum_{l, m \in \Sigma_{r}} v_{l, m} F(l, m)$.

The functions $\mathcal{G}_{M}^{+}$and $\mathcal{G}^{-}$are continuous. Indeed, for fixed $u$, if the set $\widetilde{V} \subset$ $\mathcal{M}_{1}^{*}\left(\Sigma_{r}\right)$ is a neighbourhood of the set $\left\{v \in \mathcal{M}_{1}^{*}\left(\Sigma_{r}\right): \bar{v}=u\right\}$, there exists a neighbourhood $\widetilde{U}$ of $u$ with $\left\{v \in \mathcal{M}_{1}^{*}\left(\Sigma_{r}\right): \bar{v}=\tilde{u}\right\} \subset \widetilde{V}$ for all $\tilde{u} \in \widetilde{U}$. Together with the obvious continuity of $\widetilde{I}_{u}^{2}(v)$ in both arguments $u$ and $v$ and of $v \mapsto\langle v, F\rangle$ this implies continuity of $\mathcal{G}_{M}^{+}$and $\mathcal{G}^{-}$.

Introduce the empirical measure of the vector $\sigma^{i}=\left(\sigma_{1}^{i}, \ldots, \sigma_{\lambda_{i} m}^{i}\right) \in \Sigma_{r}^{\lambda_{i} m}$ of length $\lambda_{i} m$ by putting

$$
L_{\sigma^{i}, \lambda_{i} m}=\frac{1}{\lambda_{i} m} \sum_{j=1}^{\lambda_{i} m} \delta_{\sigma_{j}^{i}} \in \mathcal{M}_{1}\left(\Sigma_{r}\right),
$$

and the global empirical measure of $\sigma=\left(\sigma^{1}, \ldots, \sigma^{n}\right) \in \Sigma_{r}^{m}$ by

$$
L_{\sigma, m}=\frac{1}{m} \sum_{i=1}^{n} \sum_{j=1}^{\lambda_{i} m} \delta_{\sigma_{j}^{i}}=\sum_{i=1}^{n} \lambda_{i} L_{\sigma^{i}, \lambda_{i} m} \in \mathcal{M}_{1}\left(\Sigma_{r}\right) .
$$


By [KM02, Lemma 3.5], for any $M>0$, uniformly in $y \in U^{m}$, as $m \uparrow \infty$,

$$
\begin{aligned}
\Phi_{m, M}(y) & \leq e^{o(m)} \exp \left(-m \mathcal{G}_{M}^{+}\left(L_{\pi y, m}\right)\right), \\
\Phi_{m}(y) & \geq e^{o(m)} \exp \left(-m \mathcal{G}^{-}\left(L_{\pi y, m}\right)\right) .
\end{aligned}
$$

Now we go back to the integral on the right-hand side of (3.13) and rewrite the integral over $B^{m}$ (which we have replaced by the integral over $U^{m}$ ) as an integral over the partition sets $U \sigma_{j}^{i}$ with $i \in\{1, \ldots, n\}$ and $j \in\left\{1, \ldots, \lambda_{i} m\right\}$ and sum over all $\sigma_{j}^{i} \in \Sigma_{r}$.

Note that, for any $\sigma \in \Sigma_{r}^{m}$, the map $y \mapsto L_{\pi y, m}=L_{\sigma, m}$ is constant on the set of $y \in U^{m}$ satisfying $y_{j}^{i} \in U_{\sigma_{j}^{i}}$, where we again organise $\sigma \in \Sigma_{r}^{m}$ as $\sigma=\left(\sigma_{j}^{i}: j=\right.$ $\left.1, \ldots, \lambda_{i} m, i=1, \ldots, n\right)$. Hence,

$$
\begin{array}{r}
\int_{B^{m}} d y\left(\Phi_{m, M}(y)\right)^{p} \prod_{i=1}^{n} \prod_{j=1}^{\lambda_{i} m} \varphi_{i}\left(y_{j}^{i}\right) \leq \sum_{\sigma \in \Sigma_{r}^{m}} e^{-p m \mathcal{G}_{M}^{+}\left(L_{\sigma, m}\right)} \prod_{i=1}^{n} \prod_{j=1}^{\lambda_{i} m} \int_{U_{\sigma_{j}^{i}}} \varphi_{i}\left(y_{j}^{i}\right) d y_{j}^{i} \\
=\sum_{\sigma \in \Sigma_{r}^{m}} e^{-p m \mathcal{G}_{M}^{+}\left(L_{\sigma, m}\right)} \prod_{i=1}^{n} \exp \left(m \lambda_{i}\left\langle L_{\sigma^{i}, m}, \log \int_{U} \varphi_{i}\right\rangle\right) .
\end{array}
$$

Analogously, we have a lower bound for the left-hand side with $\Phi_{m, M}$ replaced by $\Phi_{m}$ in terms of the right-hand side with $\mathcal{G}_{M}^{+}$replaced by $\mathcal{G}^{-}$. We rewrite the sum over $\sigma \in \Sigma_{r}^{m}$ as $n$ sums over probability measures $u_{i} \in \mathcal{M}_{1}\left(\Sigma_{r}\right)$ and count the numbers of $\sigma^{i} \in \Sigma_{r}^{\lambda_{i} m}$ such that $u_{i}$ is the empirical measure of $\sigma^{i}$,

$$
\begin{aligned}
& \int_{B^{m}} d y\left(\Phi_{m, M}(y)\right)^{p} \prod_{i=1}^{n} \prod_{j=1}^{\lambda_{i} m} \varphi_{i}\left(y_{j}^{i}\right) \\
& \leq \sum_{\substack{u_{i} \in \mathcal{M}_{1}^{\left(m \lambda_{i}\right)}\left(\Sigma_{r}\right) \\
\forall i=1, \ldots, n}} e^{-m p \mathcal{G}_{M}^{+}\left(\sum_{i=1}^{n} \lambda_{i} u_{i}\right)}\left[\prod_{i=1}^{n} \#\left\{\sigma^{i} \in \Sigma_{r}^{\lambda_{i} m}: u_{i}=L_{\sigma^{i}, m}\right\}\right] \\
& \times\left[\prod_{i=1}^{n} e^{m \lambda_{i}\left\langle u_{i}, \log \int_{U .} \varphi_{i}\right\rangle}\right]
\end{aligned}
$$

where $\mathcal{M}_{1}^{\left(m \lambda_{i}\right)}\left(\Sigma_{r}\right)$ is the set of those $u_{i}$ such that $m \lambda_{i} u_{i}(l)$ is an integer for any $l \in \Sigma_{r}$. By Stirling's formula, the $i$ th counting factor on the right is equal to $e^{o(m)} \exp \left(-m \lambda_{i} \sum_{l \in \Sigma_{r}} u_{i}(l) \log u_{i}(l)\right)$, uniformly in $u_{i} \in \mathcal{M}_{1}^{\left(m \lambda_{i}\right)}\left(\Sigma_{r}\right)$ and in $\lambda \in \mathbb{S}_{n}$. Indeed, choose $0<c<C$ such that $c \leq n ! /\left[\left(\frac{n}{e}\right)^{-n} n^{-1 / 2}\right] \leq C$ for any $n \in \mathbb{N}$, and estimate, for $\lambda_{i}>0$,

$$
\begin{aligned}
\#\left\{\sigma^{i} \in \Sigma_{r}^{\lambda_{i} m}:\right. & \left.u_{i}=L_{\sigma^{i}, m}\right\}=\frac{\left(\lambda_{i} m\right) !}{\prod_{l: u_{i}(l)>0}\left(u_{i}(l) \lambda_{i} m\right) !} \\
& \leq \exp \left\{-m \lambda_{i} \sum_{l} u_{i}(l) \log u_{i}(l)\right\} C c^{-r} \sqrt{\frac{\lambda_{i}}{\prod_{l: u_{i}(l)>0}\left(u_{i}(l) \lambda_{i}\right)}} .
\end{aligned}
$$

Now use that $u_{i}(l) \lambda_{i} \geq \frac{1}{m}$ if $u_{i}(l)>0$. The lower bound is derived in a similar way. 
Note that the cardinality of $\mathcal{M}_{1}^{\left(m \lambda_{i}\right)}\left(\Sigma_{r}\right)$ is polynomial in $m$, uniformly in $\lambda \in \mathbb{S}_{n}$. Hence, we obtain

$$
\underset{m \uparrow \infty}{\limsup } \sup _{\lambda \in \mathbb{S}_{n}}\left[\frac{1}{m} \log \left(\int_{B^{m}} d y\left(\Phi_{m, M}(y)\right)^{p} \prod_{i=1}^{n} \prod_{j=1}^{\lambda_{i} m} \varphi_{i}\left(y_{j}^{i}\right)\right)-\widetilde{\mathfrak{H}}_{M}^{+}(\phi, \lambda)\right] \leq 0
$$

where we recall that $\varphi_{i}=\phi_{i}^{2 p}$ and introduce

$$
\widetilde{\mathfrak{H}}_{M}^{+}(\phi, \lambda)=-\inf _{u_{1}, \ldots, u_{n} \in \mathcal{M}_{1}\left(\Sigma_{r}\right)}\left[p \mathcal{G}_{M}^{+}\left(\sum_{i=1}^{n} \lambda_{i} u_{i}\right)+\sum_{i=1}^{n} \lambda_{i} H\left(u \mid \int_{U} \varphi_{i}\right)\right] .
$$

It is easy to see that the map $\widetilde{\mathfrak{H}}_{M}^{+}(\phi, \cdot)$ is continuous on the simplex $\mathbb{S}_{n}$. Indeed, the family of mappings

$$
\lambda \mapsto p \mathcal{G}_{M}^{+}\left(\sum_{i=1}^{n} \lambda_{i} u_{i}\right)+\sum_{i=1}^{n} \lambda_{i} H\left(u_{i} \mid \int_{U .} \varphi_{i}\right), \quad \text { for } u_{1}, \ldots, u_{n} \in \mathcal{M}_{1}\left(\Sigma_{r}\right),
$$

is uniformly equicontinuous on $\mathbb{S}_{n}$, since $\mathcal{G}_{M}^{+}$is uniformly continuous on $\mathcal{M}_{1}\left(\Sigma_{r}\right)$, and the map $u \mapsto H\left(u \mid \int_{U} \varphi_{i}\right)$ is bounded and continuous for every $i$.

Analogously to (3.22), we have, for any $\lambda \in \mathbb{S}_{n}$,

$$
\liminf _{k \uparrow \infty} \frac{1}{k} \log \left(\int_{B^{k}} d y\left(\Phi_{k}(y)\right)^{p} \prod_{i=1}^{n} \prod_{j=1}^{\lambda_{i} k} \varphi_{i}\left(y_{j}^{i}\right)\right) \geq \widetilde{\mathfrak{H}}^{-}(\phi, \lambda),
$$

where $\widetilde{\mathfrak{H}}^{-}(\phi, \lambda)$ is defined as $\widetilde{\mathfrak{H}}_{M}^{+}(\phi, \lambda)$ with $\mathcal{G}_{M}^{+}$replaced by $\mathcal{G}^{-}$. Like $\widetilde{\mathfrak{H}}_{M}^{+}(\phi, \cdot)$, the function $\widetilde{\mathfrak{H}}^{-}(\phi, \cdot)$ is also continuous on $\mathbb{S}_{n}$.

Now we determine the fineness of the partition $\left(U_{1}, \ldots, U_{r}\right)$ of the set $U$.

Lemma 3.3 (Choice of the partition). Fix $M>0$ and any $\delta>0$.

(1) For any $\lambda \in \mathbb{S}_{n}$, the partition $\left(U_{1}, \ldots, U_{r}\right)$ of the set $U$ may be chosen so fine that

$$
\widetilde{\mathfrak{H}}^{-}(\phi, \lambda) \geq \mathfrak{H}(\phi, \lambda)-\delta
$$

(2) If each $\phi_{i}$ is bounded away from zero on its support, then the partition $\left(U_{1}, \ldots, U_{r}\right)$ of the set $U$ may be chosen so fine that, for any $\lambda \in \mathbb{S}_{n}$,

$$
\widetilde{\mathfrak{H}}_{M}^{+}(\phi, \lambda) \leq \mathfrak{H}(\phi, \lambda)+\delta .
$$

(3) For any $H \subset \mathcal{M}_{1}(U)$ and $\delta>0$ the partition $\left(U_{1}, \ldots, U_{r}\right)$ of the set $U$ may be chosen so fine that

$$
-\inf _{\mu \in H}\left\{\widetilde{I}(\pi \mu)+p \mathcal{G}_{M}^{+}(\pi \mu)\right\} \leq-\inf _{\mu \in H[\delta]}\{I(\mu)+p \mathcal{G}(\mu)\}+\delta
$$

Proof. We first prove (i). Choose approximate minimisers $\mu_{1}^{*}, \ldots, \mu_{n}^{*} \in \mathcal{M}_{1}(U)$ in (2.6) and an approximate minimiser $\nu^{*} \in \mathcal{M}_{1}^{*}\left(B^{2}\right)$ in the definition (2.5) of $\mathcal{G}$ (satisfying $\bar{\nu}^{*}=\sum_{i=1}^{n} \lambda_{i} \mu_{i}^{*}$ ) such that

$$
-\mathfrak{H}(\phi, \lambda) \geq \sum_{i=1}^{n} \lambda_{i} H\left(\mu_{i}^{*} \mid \varphi_{i}\right)+p\left(I_{\bar{\nu}^{*}}^{2}\left(\nu^{*}\right)-\left\langle\nu^{*}, \log G\right\rangle\right)-\frac{\delta}{2} .
$$

We now use Jensen's inequality to show that, for any partition and for any $\mu$ resp. $\nu$, we have

$$
H\left(\mu \mid \varphi_{i}\right) \geq H\left(\pi \mu \mid \int_{U .} \varphi_{i}\right) \quad \text { and } \quad I_{\bar{\nu}}^{2}(\nu) \geq \widetilde{I}_{\pi \bar{\nu}}^{2}(\pi \nu)
$$


To prove this, abbreviate $f(y)=y \log y$ and note that

$$
H\left(\mu \mid \varphi_{i}\right)=\sum_{l} \int_{U_{l}} \varphi_{i}(x) d x \int_{U_{l}} \frac{d x \varphi_{i}(x)}{\int_{U_{l}} \varphi_{i}(x) d x} f\left(\frac{\mu(d x) / d x}{\varphi_{i}(x)}\right) .
$$

Now use Jensen's inequality for the convex function $f$ and summarise to arrive at the first inequality in (3.25). The other one is proved analogously, noting that $I_{\bar{\nu}}^{2}(\nu)=\langle\bar{\nu} \otimes \bar{\nu}, f \circ(d \nu / d(\bar{\nu} \otimes \bar{\nu}))\rangle$.

For $\nu^{*}$ fixed above, we may choose the partition $\left(U_{1}, \ldots, U_{r}\right)$ of $U$ so fine that $\left\langle\nu^{*}, \log G\right\rangle \leq\left\langle\pi \nu^{*}, \log G^{-}\right\rangle+\delta /(2 p)$. This can be seen by choosing $N$ so large that $\langle\nu, \log G\rangle-\left\langle\nu, \log G_{N}\right\rangle<\delta /(4 p)$ and using uniform continuity of $\log G_{N}$ on $U^{2}$ to split the domain of integration into partition sets on which the variation of $\log G_{N}$ is less than $\delta /(4 p)$. Using this and (3.25) in (3.24), we arrive at

$$
\begin{aligned}
-\mathfrak{H}(\phi, \lambda) & \geq \sum_{i=1}^{n} \lambda_{i} H\left(\pi \mu_{i}^{*} \mid \int_{U .} \varphi_{i}\right)+p\left(\widetilde{I}_{\pi \bar{\nu}^{*}}^{2}\left(\pi \nu^{*}\right)-\left\langle\pi \nu^{*}, \log G^{-}\right\rangle\right)-\delta \\
& \geq \inf _{u_{1}, \ldots, u_{n} \in \mathcal{M}_{1}\left(\Sigma_{r}\right)}\left[\sum_{i=1}^{n} \lambda_{i} H\left(u \mid \int_{U} \varphi_{i}\right)+p \mathcal{G}^{-}\left(\sum_{i=1}^{n} \lambda_{i} u_{i}\right)\right]-\delta \\
& =-\widetilde{\mathfrak{H}}^{-}(\phi, \lambda)-\delta,
\end{aligned}
$$

which finishes the proof of (i).

Now we prove (ii). We choose the partition so fine that

$$
\left|\log G_{M}^{+}(\pi x, \pi y)-\log G_{M}(x, y)\right|<\frac{\delta}{2 p}, \quad \text { for all } x, y \in U
$$

and

$$
\left|\log \widetilde{\varphi}_{i}(x)-\log \varphi_{i}(x)\right|<\frac{\delta}{2}, \quad \text { for all } i \in\{1, \ldots, n\}, x \in \operatorname{supp}\left(\varphi_{i}\right),
$$

where $\widetilde{\varphi}_{i}(x)=\sum_{l=1}^{r} \operatorname{Leb}\left(U_{l}\right)^{-1} 1_{U_{l}}(x) \int_{U_{l}} \varphi_{i}$ is a discrete approximation to $\varphi_{i}$. This choice is possible since the functions $\log G_{M}$ and $\log \varphi_{i}$ are bounded and measurable on $U^{2}$ resp. on $\operatorname{supp}\left(\varphi_{i}\right)$. To every $\mu \in \mathcal{M}_{1}(U)$ we associate a $\widetilde{\mu} \in \mathcal{M}_{1}(U)$ with constant density on the partition sets and $\pi \mu=\pi \widetilde{\mu}$. Note that $H\left(\pi \mu \mid \int_{U} \varphi_{i}\right)=$ $H\left(\widetilde{\mu} \mid \widetilde{\varphi}_{i}\right)$ for any $i \in\{1, \ldots, n\}$ and any $\mu \in \mathcal{M}_{1}(U)$. Furthermore, we easily derive from (3.26) resp. from (3.27) that

$$
\mathcal{G}_{M}^{+}(\pi \mu) \geq \mathcal{G}(\widetilde{\mu})-\frac{\delta}{2 p} \quad \text { and } \quad H\left(\pi \mu \mid \widetilde{\varphi}_{i}\right) \geq H\left(\widetilde{\mu} \mid \varphi_{i}\right)-\frac{\delta}{2},
$$

for any $\mu \in \mathcal{M}_{1}(U)$ and any $i \in\{1, \ldots, n\}$. Hence, we obtain, for any $\lambda \in \mathbb{S}_{n}$,

$$
\begin{aligned}
\mathfrak{H}_{M}^{+}(\phi, \lambda) & =-\inf _{\mu_{1}, \ldots, \mu_{n} \in \mathcal{M}_{1}(U)}\left(p \mathcal{G}_{M}^{+}\left(\pi\left(\sum_{i=1}^{n} \lambda_{i} \mu_{i}\right)\right)+\sum_{i=1}^{n} \lambda_{i} H\left(\pi \mu_{i} \mid \int_{U .} \varphi_{i}\right)\right) \\
& \leq-\inf _{\mu_{1}, \ldots, \mu_{n} \in \mathcal{M}_{1}(U)}\left(p \mathcal{G}\left(\sum_{i=1}^{n} \lambda_{i} \widetilde{\mu}_{i}\right)+\sum_{i=1}^{n} \lambda_{i} H\left(\widetilde{\mu}_{i} \mid \varphi_{i}\right)\right)+\delta \\
& \leq-\inf _{\mu_{1}, \ldots, \mu_{n} \in \mathcal{M}_{1}(U)}\left(p \mathcal{G}\left(\sum_{i=1}^{n} \lambda_{i} \mu_{i}\right)+\sum_{i=1}^{n} \lambda_{i} H\left(\mu_{i} \mid \varphi_{i}\right)\right)+\delta \\
& =\mathfrak{H}(\phi, \lambda)+\delta .
\end{aligned}
$$


Finally we prove (iii). We choose the partition so fine that (3.26) holds and such that $\mu \in H, \pi \mu=\pi \widetilde{\mu}$ imply $\widetilde{\mu} \in H[\delta]$. As in the proof of (ii) we associate to any $\mu \in H$ a measure $\widetilde{\mu} \in \mathcal{M}_{1}(U)$ with constant density on the partition sets and $\pi \mu=\pi \widetilde{\mu}$. In particular, this implies $\widetilde{\mu} \in H[\delta]$. Since $\widetilde{I}(\pi \mu)=I(\widetilde{\mu})$, the statement follows as above.

We now complete the proof of Proposition 2.1. For part (i), it suffices to combine (3.2), (3.23) and Lemma 3.3(i). For the proof of part (ii) let $\delta>0$ be small. By (3.2) and Lemma 3.2(i) we can choose $M>0$ such that, for all $\lambda \in \mathbb{S}_{n}$,

$$
\mathbb{E}\left[\frac{1}{k !^{p}} \prod_{i=1}^{n}\left\langle\varphi_{i}, \ell\right\rangle^{k \lambda_{i}}\right] \leq \delta^{k}+(1+\delta)^{k} \sum_{m=\lceil k(1-\delta)\rceil}^{k} \int_{B^{m}} d y\left(\Phi_{m, M}(y)\right)^{p} \prod_{i=1}^{n} \prod_{j=1}^{\lambda_{i} m} \varphi_{i}\left(y_{j}^{i}\right) .
$$

The right-hand side can further be estimated, using (3.22) and Lemma 3.3(ii), for sufficiently large $k$ and all $\lambda \in \mathbb{S}_{n}$, by

$$
\delta^{k}+(1+\delta)^{k} \sum_{m=\lceil k(1-\delta)\rceil}^{k} e^{m(\mathfrak{H}(\phi, \lambda)+2 \delta)} .
$$

Now we argue that $\mathfrak{H}(\phi, \lambda)$ is bounded from below in $\lambda \in \mathbb{S}_{n}$. Indeed, in (2.6), we get a lower bound by choosing $\mu_{i}(d x)=c_{i} \phi^{2 p}(x) d x$, and noting that $\mathcal{G}$ is bounded from above on $\mathcal{M}_{1}(U)$. From this the proof of Proposition 2.1(2) readily follows.

For the proof of Proposition 2.1(iii) let $\delta>0$ be small. By (3.3) and by Lemma 3.2(ii) we can choose $M>0$ such that

$$
\mathbb{E}\left[\frac{1}{k !^{p}} \ell(U)^{k} 1_{\{L \in H\}}\right] \leq \delta^{k}+(1+\delta)^{k} \sum_{m=\lceil k(1-\delta)\rceil}^{k} \int_{U^{m}} d y\left(\Phi_{m, M}(y)\right)^{p} 1_{\left\{L_{y, m} \in H[\delta]\right\}} .
$$

Now we use (3.20) and note that $L_{\pi y, m}=\pi L_{y, m}$ to obtain

$$
\limsup _{m \uparrow \infty} \frac{1}{m} \log \left(\int_{U^{m}} d y\left(\Phi_{m, M}(y)\right)^{p} 1_{\left\{L_{y, m} \in H[\delta]\right\}}\right) \leq-\inf _{\mu \in H[\delta]}\left\{\widetilde{I}(\pi \mu)+p \mathcal{G}_{M}^{+}(\pi \mu)\right\} .
$$

From here one can finish the proof of Proposition 2.1(iii) by an application of Lemma 3.3(iii).

\section{IDENTIFICATION OF THE VARIATIONAL FORMULA}

In this section we prove Proposition 2.3. This is done in two steps. In Section 4.1 we identify $W(\phi)$ in terms of a variational problem involving energies of measures with respect to the Green operator on $B$. In Section 4.2 this formula is related to the variational formula (1.7) for $\Theta(\phi)$ in that section, and this completes the proof of Proposition 2.3

4.1. Identification of $\boldsymbol{W}(\phi)$ in terms of energies of measures. Recall the definition of the Green function $G$ from Section 2.1 and define the associated operator $\mathfrak{A}$ by

$$
\mathfrak{A} g(x)=\int G(x, y) g(y) d y \quad \text { and } \quad \mathfrak{A} \mu(x)=\int G(x, y) \mu(d y) .
$$


We introduce the energy of a measure $\mu$ on $B$,

$$
\|\mu\|_{\mathrm{E}}^{2}=\langle\mu, \mathfrak{A} \mu\rangle=\int_{B} \int_{B} \mu(d x) G(x, y) \mu(d y),
$$

and we write $\|g\|_{E}=\|\mu\|_{E}$ if $\mu=g d x$.

Let $\phi=\left(\phi_{1}, \ldots, \phi_{n}\right)$ be a family of nonnegative, bounded measurable functions on $B$ having compact supports. The main object of this section is the variational formula

$$
\begin{aligned}
\rho(\phi)=\rho\left(\phi_{1}, \ldots, \phi_{n}\right)= & \sup \left\{\left\|\sum_{i=1}^{n} \sqrt{\lambda_{i}} g_{i}^{2 p-1} \phi_{i}\right\|_{\mathrm{E}}^{2}:\right. \\
& \left.\lambda \in \mathbb{S}_{n}, g_{i} \in L^{2 p}(B),\left\|g_{i}\right\|_{2 p}=1 \text { for } i=1, \ldots, n\right\} .
\end{aligned}
$$

We first show that maximisers exist for this variational problem, and we derive the variational equations.

Lemma 4.1 (Analysis of $\rho(\phi))$. Let $\phi=\left(\phi_{1}, \ldots, \phi_{n}\right)$ be a family of nonnegative, bounded measurable functions on $B$ with compact supports. Then there exist $\lambda \in \mathbb{S}_{n}$ and $g_{1}, \ldots, g_{n} \in L^{2 p}(B)$ with $\left\|g_{i}\right\|_{2 p}=1$ such that

$$
\rho(\phi)=\left\|\sum_{i=1}^{n} \sqrt{\lambda_{i}} g_{i}^{2 p-1} \phi_{i}\right\|_{\mathrm{E}}^{2}
$$

and

$$
\sqrt{\lambda_{i}} \rho(\phi) g_{i}=\phi_{i} \mathfrak{A}\left(\sum_{j=1}^{n} \sqrt{\lambda_{j}} g_{j}^{2 p-1} \phi_{j}\right), \quad \text { for all } i=1, \ldots, n .
$$

Proof. We may assume that the supports of the $\phi_{i}$ are not empty. Then it is clear that in (4.2) we may add the conditions $g_{i} \geq 0$ and $\operatorname{supp}\left(g_{i}\right) \subset U$ for all $i=1, \ldots, n$, where $U=\bigcup_{i=1}^{n} \operatorname{supp}\left(\phi_{i}\right)$ denotes the union of the supports of $\phi_{1}, \ldots, \phi_{n}$, which is a compact subset of $B$. Furthermore, we may relax the condition $\left\|g_{i}\right\|_{2 p}=1$ to the condition $\left\|g_{i}\right\|_{2 p} \leq 1$. It is convenient to substitute $f_{i}=g_{i}^{2 p-1}$ and to rewrite (4.2) as

$$
\rho(\phi)=\sup \left\{\left\|\sum_{i=1}^{n} \sqrt{\lambda_{i}} f_{i} \phi_{i}\right\|_{\mathrm{E}}^{2}: \lambda \in \mathbb{S}_{n}, f_{1}, \ldots, f_{n} \in K_{1}\right\},
$$

where

$$
K_{M}=\left\{f \in L^{1}(U): f \geq 0,\|f\| \leq M\right\}, \quad \text { for } M>0,
$$

and $\|\cdot\|=\|\cdot\|_{2 p /(2 p-1)}$. As a first step, we argue that maximisers exist for the problem in (4.5). In the proof of [KM02, Lemma 4.3] we showed that $K_{1}$ is weakly compact in $L^{1}(U)$ and that the map $f \mapsto\|f\|_{\mathrm{E}}^{2}$ is upper semicontinuous on $K_{1}$ in the weak topology on $L^{1}(U)$. Certainly, these two statements also hold for $K_{M}$ for any $M>0$. Since the set $\mathbb{S}_{n} \times K_{1}^{n}$ is also compact and since the map

$$
\mathbb{S}_{n} \times K_{1}^{n} \ni\left(\lambda, f_{1}, \ldots, f_{n}\right) \mapsto \sum_{i=1}^{n} \sqrt{\lambda_{i}} f_{i} \phi_{i} \in K_{M}
$$

(here $M>0$ is suitably chosen, only dependent on $\phi_{1}, \ldots, \phi_{n}$ ) is continuous in the product topology, the existence of maximisers in (4.5) follows. We denote them by $\lambda \in \mathbb{S}_{n}$ and $f_{1}, \ldots, f_{n} \in K_{1}$. It is clear that $\left\|f_{i}\right\|=1$ and $\operatorname{supp}\left(f_{i}\right) \subset \operatorname{supp}\left(\phi_{i}\right)$ for all $i=1, \ldots, n$. 
The second step is to show that $\lambda_{i}>0$ and that $f_{i}$ is bounded away from 0 on any set where $\phi_{i}$ is bounded from zero, for any $i=1, \ldots, n$. Let us first prove the first of these two statements. Assume the contrary, i.e., $\lambda_{1}=0$, say. Then we may assume that $f_{1} \phi_{1}$ is not almost everywhere equal to zero. There is an $i \in\{2, \ldots, n\}$ such that $\sqrt{\lambda_{i}} f_{i} \phi_{i}$ is not almost everywhere equal to zero. For definiteness, we assume that $\lambda_{2}>0$ and that $f_{2} \phi_{2}$ is not trivial. With some $\delta>0$, we define $\tilde{\lambda} \in \mathbb{S}_{n}$ by

$$
\tilde{\lambda}_{j}= \begin{cases}\delta, & \text { if } j=1 \\ \lambda_{2}-\delta, & \text { if } j=2 \\ \lambda_{j}, & \text { otherwise }\end{cases}
$$

The idea is to pick $\delta>0$ so small that $\left\|h_{\tilde{\lambda}}\right\|_{\mathrm{E}}^{2}>\left\|h_{\lambda}\right\|_{\mathrm{E}}^{2}$, where $h_{\lambda}=\sum_{j=1}^{n} \sqrt{\lambda_{j}} f_{j} \phi_{j}$. This would contradict the maximality of $\lambda$ and therefore prove the first assertion. We calculate

$$
\begin{aligned}
&\left\|h_{\tilde{\lambda}}\right\|_{\mathrm{E}}^{2}-\left\|h_{\lambda}\right\|_{\mathrm{E}}^{2}=\sum_{i, j=1}^{n}\left(\sqrt{\tilde{\lambda}_{i} \tilde{\lambda}_{j}}-\sqrt{\lambda_{i} \lambda_{j}}\right)\left\langle f_{i} \phi_{i}, \mathfrak{A}\left(f_{j} \phi_{j}\right)\right\rangle \\
&=\delta\left\langle f_{1} \phi_{1}, \mathfrak{A}\left(f_{1} \phi_{1}\right)\right\rangle+2 \sqrt{\delta} \sqrt{\lambda_{2}-\delta}\left\langle f_{1} \phi_{1}, \mathfrak{A}\left(f_{2} \phi_{2}\right)\right\rangle \\
& \quad+2 \sqrt{\delta} \sum_{j=3}^{n} \sqrt{\lambda_{j}}\left\langle f_{1} \phi_{1}, \mathfrak{A}\left(f_{j} \phi_{j}\right)\right\rangle \\
& \quad+2\left(\sqrt{\lambda_{2}-\delta}-\sqrt{\lambda_{2}}\right) \sum_{j=3}^{n} \sqrt{\lambda_{j}}\left\langle f_{2} \phi_{2}, \mathfrak{A}\left(f_{j} \phi_{j}\right)\right\rangle-\delta\left\langle f_{2} \phi_{2}, \mathfrak{A}\left(f_{2} \phi_{2}\right)\right\rangle \\
& \geq \sqrt{\delta}\left(c_{1}\left\langle f_{1} \phi_{1}, \mathfrak{A}\left(f_{2} \phi_{2}\right)\right\rangle-c_{2} \sqrt{\delta}\right),
\end{aligned}
$$

for positive constants $c_{1}, c_{2}$, not depending on $\delta$. Since $f_{1} \phi_{1}$ and $f_{2} \phi_{2}$ are nonnegative and not trivial, and since $G$ is bounded away from zero on $U^{2}$, it is clear that the right-hand side of (4.6) is positive for sufficiently small $\delta>0$. This contradicts the maximality of $\lambda$. Hence, $\lambda_{i}>0$ for all $i \in\{1, \ldots, n\}$.

Now we fix a small $\delta>0$ and prove that every $f_{i}$ is essentially bounded away from 0 on $\left\{\phi_{i}>\delta\right\}$. Abbreviate $U_{1}=\left\{\phi_{1}>\delta\right\}$ and assume for contradiction that $\left|\left\{f_{1} \leq \varepsilon\right\} \cap U_{1}\right|>0$ for all $\varepsilon>0$. Pick some $c>0$ such that $\left|\left\{f_{1}>c\right\} \cap U_{1}\right|>0$. With some $a, b>0$, we define $\tilde{f}_{1}: U \rightarrow[0, \infty)$ by

$$
\tilde{f}_{1}(x)= \begin{cases}f_{1}(x)+a, & \text { if } f_{1}(x) \leq \varepsilon \\ f_{1}(x)-b, & \text { if } f_{1}(x) \geq c \\ f_{1}(x), & \text { otherwise }\end{cases}
$$

The idea is to pick $a, b>0$ in such a way that $\left\|\tilde{f}_{1}\right\|=1$ but $\left\|\tilde{h}_{\lambda}\right\|_{\mathrm{E}}^{2}>\left\|h_{\lambda}\right\|_{\mathrm{E}}^{2}$, where $h_{\lambda}=\sum_{j=1}^{n} \sqrt{\lambda_{j}} f_{j} \phi_{j}$, and $\tilde{h}_{\lambda}$ is defined analogously with $f_{1}$ replaced by $\tilde{f}_{1}$. This would contradict the maximality of $f_{1}, \ldots, f_{n}$ and therefore prove the assertion.

For notational convenience, we put $\tilde{f}_{i}=f_{i}$ for $i \geq 2$. Abbreviate $\eta=1 /(2 p-1)$. For every sufficiently small $a$ and $\varepsilon>0$, we can find $b \in(0, c / 2)$ such that $\left\|\tilde{f}_{1}\right\|=1$. 
This implies

$$
\begin{aligned}
& 0=\left\|\tilde{f}_{1}\right\|^{1+\eta}-\left\|f_{1}\right\|^{1+\eta} \\
& =\int_{\{f \leq \varepsilon\} \cap U_{1}}\left[\left(f_{1}(x)+a\right)^{1+\eta}-f_{1}(x)^{1+\eta}\right] d x \\
& \quad+\int_{\{f \geq c\} \cap U_{1}}\left[\left(f_{1}(x)-b\right)^{1+\eta}-f_{1}(x)^{1+\eta}\right] d x .
\end{aligned}
$$

Hence, for some constant $C>0$ depending neither on $a$ nor on $\varepsilon$, we have $b \leq$ $C a(a+\varepsilon)^{\eta}\left|U_{1} \cap\left\{f_{1} \leq \varepsilon\right\}\right|$. Now we calculate

$$
\begin{aligned}
& \left\|h_{\tilde{\lambda}}\right\|_{\mathrm{E}}^{2}-\left\|h_{\lambda}\right\|_{\mathrm{E}}^{2}=\sum_{i, j=1}^{n} \sqrt{\lambda_{i} \lambda_{j}}\left(\left\langle\tilde{f}_{i} \phi_{i}, \mathfrak{A}\left(\tilde{f}_{j} \phi_{j}\right)\right\rangle-\left\langle f_{i} \phi_{i}, \mathfrak{A}\left(f_{j} \phi_{j}\right)\right\rangle\right) \\
& =\lambda_{1} \int_{U_{1} \cap\left\{f_{1} \leq \varepsilon\right\}} \int_{U_{1} \cap\left\{f_{1} \leq \varepsilon\right\}} d x d y G(x, y) \phi_{1}(x) \phi_{1}(y) \\
& \times \\
& +\lambda_{1} \int_{U_{1} \cap\left\{f_{1} \geq c\right\}} \int_{U_{1} \cap\left\{f_{1} \geq c\right\}} d x d y G(x, y) \phi_{1}(x) \phi_{1}(y) \\
& \times \\
& \left.+2 \lambda_{1} \int_{U_{1} \cap\left\{f_{1} \leq \varepsilon\right\}} \int_{U_{1} \cap\left\{f_{1} \geq c\right\}} d\left(f_{1}(x)-b\right)\left(f_{1}(y)-b\right)-f_{1}(x) f_{1}(y)\right] \\
& \times\left[\left(f_{1}(x)+a\right)\left(f_{1}(y)-b\right)-f_{1}(x) f_{1}(y)\right] \\
& +2 \sqrt{\lambda_{1}} \sum_{j=2}^{n} \sqrt{\lambda_{j}} \int_{U_{1} \cap\left\{f_{1} \leq \varepsilon\right\}} \int_{U_{j}} d x d y G(x, y) \phi_{1}(x) \phi_{j}(y) \\
& \times\left[\left(f_{1}(x)+a\right)-f_{1}(x)\right] f_{j}(y) \\
& -2 \sqrt{\lambda_{1}} \sum_{j=2}^{n} \sqrt{\lambda_{j}} \int_{U_{1} \cap\left\{f_{1} \geq c\right\}} \int_{U_{j}} d x d y G(x, y) \phi_{1}(x) \phi_{j}(y) \\
& \times\left[\left(f_{1}(x)-b\right)-f_{1}(x)\right] f_{j}(y) \\
& \geq a C_{1}\left|U_{1} \cap\left\{f_{1} \leq \varepsilon\right\}\right|-b C_{2},
\end{aligned}
$$

for some constants $C_{1}>0$ and $C_{2}>0$, depending neither on $a$ nor on $b$. From the bound on $b$, we see that the right-hand side of (4.7) is positive for $a>0$ and $b>0$ sufficiently small, if $\varepsilon>0$ is sufficiently small. This contradicts the maximality of $f_{1}, \ldots, f_{n}$. Hence, every $f_{i}$ is essentially bounded away from zero on sets of the form $\left\{\phi_{i}>\delta\right\}$.

The third and last step is a standard application of variational techniques to derive the variational equation in (4.4) for the maximisers $\lambda \in \mathbb{S}_{n}$ and $f_{1}, \ldots, f_{n} \in$ $K$. It is convenient to substitute

$$
r_{i}=g_{i}^{2 p}=f_{i}^{2 p /(2 p-1)} \text { for } i=1, \ldots, n ;
$$

then $r_{i}$ is normalized in $L^{1}(U)$-sense. For any family of test functions $\varphi_{i}:\left\{\phi_{i}>\right.$ $\delta\} \rightarrow \mathbb{R}$ satisfying $\int \varphi_{i}=0$ for $i=1, \ldots, n$, and for any vector $v=\left(v_{1}, \ldots, v_{n}\right)$ satisfying $\sum_{i=1}^{n} v_{i}=0$, the objects $\lambda+\varepsilon v$ and $r_{i}+\varepsilon \varphi_{i}$ are admissible for all $\varepsilon$ with 
$|\varepsilon|$ sufficiently small, and we obtain

$$
\begin{aligned}
0= & \left.\frac{d}{d \varepsilon}\right|_{\varepsilon=0}\left\|\sum_{i=1}^{n} \sqrt{\lambda_{i}+\varepsilon v_{i}}\left(r_{i}+\varepsilon \varphi_{i}\right)^{\frac{2 p-1}{2 p}} \phi_{i}\right\|_{\mathrm{E}}^{2} \\
= & \frac{2 p-1}{p} \sum_{i=1}^{n} \sqrt{\lambda_{i}}\left\langle\varphi_{i}, r_{i}^{-\frac{1}{2 p}} \phi_{i}, \mathfrak{A}\left(\sum_{j=1}^{n} \sqrt{\lambda_{j}} r_{j}^{\frac{2 p-1}{2 p}} \phi_{j}\right)\right\rangle \\
& \quad+\sum_{i=1}^{n} v_{i} \frac{1}{\sqrt{\lambda_{i}}}\left\langle r_{i}^{\frac{2 p-1}{2 p}} \phi_{i}, \mathfrak{A}\left(\sum_{j=1}^{n} \sqrt{\lambda_{j}} r_{j}^{\frac{2 p-1}{2 p}} \phi_{j}\right)\right\rangle \\
= & \frac{2 p-1}{p} \sum_{i=1}^{n} \sqrt{\lambda_{i}}\left\langle\varphi_{i}, g_{i}^{-1} \phi_{i} \mathfrak{A}\left(h_{\lambda}\right)\right\rangle+\sum_{i=1}^{n} v_{i} \frac{1}{\sqrt{\lambda_{i}}}\left\langle g_{i}^{2 p-1} \phi_{i}, \mathfrak{A}\left(h_{\lambda}\right)\right\rangle,
\end{aligned}
$$

where we put $h_{\lambda}=\sum_{j=1}^{n} \sqrt{\lambda_{j}} g_{j}^{2 p-1} \phi_{j}$. Putting $\varphi_{i}=0$ for all $i$, we obtain $C>0$ such that

$$
C \sqrt{\lambda_{i}}=\left\langle g_{i}^{2 p-1} \phi_{i}, \mathfrak{A}\left(h_{\lambda}\right)\right\rangle \text { for all } i .
$$

Multiplying this with $\sqrt{\lambda_{i}}$, summing over $i$ and using $\rho(\phi)=\left\langle h_{\lambda}, \mathfrak{A}\left(h_{\lambda}\right)\right\rangle$, it follows that $C=\rho(\phi)$. Putting $v_{i}=0$ for all $i$ in (4.8) and choosing all but one $\varphi_{j}$ equal to zero, we obtain the existence of $C_{1}, \ldots, C_{n}>0$ such that $C_{i} g_{i}=\phi_{i} \mathfrak{A}\left(h_{\lambda}\right)$ for all $i$. Multiplying the latter equality by $g_{i}^{2 p-1}$, integrating over $B$ and using (4.9), one easily obtains that $C_{i}=\rho(\phi) \sqrt{\lambda_{i}}$ for all $i$. This completes the proof of (4.4).

Now we characterise $W(\phi)$ in terms of $\rho(\phi)$. Recall the definitions (2.9) and (4.2) of $W(\phi)$ and $\rho(\phi)$, respectively.

Proposition 4.2 (Relation between $W$ and $\rho)$. Let $\phi=\left(\phi_{1}, \ldots, \phi_{n}\right)$ be a family of nonnegative, bounded measurable functions on $B$ having compact supports. Then $W(\phi)=p \log \rho(\phi)$, i.e.,

$$
\begin{gathered}
-\min _{\lambda \in \mathbb{S}_{n}} \min _{\mu_{1}, \ldots, \mu_{n} \in \mathcal{M}_{1}(B)}\left\{\sum_{i=1}^{n}\left\langle\lambda_{i} \mu_{i}, \log \left(\frac{\lambda_{i}^{p}}{\phi_{i}^{2 p}} \frac{d \mu_{i}}{d x}\right)\right\rangle+p \mathcal{G}\left(\sum_{i=1}^{n} \lambda_{i} \mu_{i}\right)\right\} \\
=p \log \max \left\{\left\|\sum_{i=1}^{n} \sqrt{\lambda_{i}} g_{i}^{2 p-1} \phi_{i}\right\|_{\mathrm{E}}^{2}:\right. \\
\left.\lambda \in \mathbb{S}_{n}, g_{i} \in L^{2 p}(B),\left\|g_{i}\right\|_{2 p}=1 \text { for } i=1, \ldots, n\right\} .
\end{gathered}
$$

An explicit one-to-one correspondence between the maximisers on the right and the minimisers on the left-hand side is given by the relation $g_{i}^{2 p}=\frac{d \mu_{i}}{d x}$ for $i=1, \ldots, n$.

Proof. In order to prove ' $\leq$ ' in (4.10), we shall show that, for any $\lambda \in \mathbb{S}_{n}$ and any $\mu_{1}, \ldots, \mu_{n} \in \mathcal{M}_{1}(B)$,

$$
-\sum_{i=1}^{n}\left\langle\lambda_{i} \mu_{i}, \log \left(\frac{\lambda_{i}^{p}}{\phi_{i}^{2 p}} \frac{d \mu_{i}}{d x}\right)\right\rangle-p \mathcal{G}\left(\sum_{i=1}^{n} \lambda_{i} \mu_{i}\right) \leq p \log \left\|h_{\lambda}\right\|_{\mathrm{E}}^{2}
$$

where we put $g_{i}^{2 p}=\frac{d \mu_{i}}{d x}$ for $i=1, \ldots, n$ and abbreviated $h_{\lambda}=\sum_{i=1}^{n} \sqrt{\lambda_{i}} g_{i}^{2 p-1} \phi_{i}$. 
Abbreviate $\mu=\sum_{i=1}^{n} \lambda_{i} \mu_{i}$ and $g^{2 p}=d \mu / d x$. Using the definition of $\mathcal{G}$, Jensen's inequality and the concavity of log, we get the following upper bound:

$$
\begin{aligned}
-\sum_{i=1}^{n}\langle & \left.\lambda_{i} \mu_{i}, \log \left(\frac{\lambda_{i}^{p}}{\phi_{i}^{2}} \frac{d \mu}{d x}\right)\right\rangle-p \mathcal{G}\left(\sum_{i=1}^{n} \lambda_{i} \mu_{i}\right) \\
= & -p\left[\sum_{i=1}^{n} \lambda_{i}\left\langle\mu_{i}, \log \frac{\lambda_{i} g_{i}^{2}}{\phi_{i}^{2}}\right\rangle+\left\langle\mu, \log \frac{h_{\lambda}}{g^{2 p}}\right\rangle\right. \\
& \left.\quad-\sup _{\nu: \bar{\nu}=\mu} \int \mu(d x) \int \frac{\nu(d x d y)}{\mu(d x)} \log \frac{g^{2 p}(x) G(x, y) h_{\lambda}(y)}{\frac{\nu(d x d y)}{d x d y}}\right] \\
\leq & -p\left[\sum_{i=1}^{n} \lambda_{i}\left\langle\mu_{i}, \log \frac{\lambda_{i} g_{i}^{2} h_{\lambda}}{\phi_{i}^{2} g^{2 p}}\right\rangle-\left\langle\mu, \log \mathfrak{A}\left(h_{\lambda}\right)\right\rangle\right] \\
= & p\left[\sum_{i=1}^{n} \lambda_{i}\left\langle g_{i}^{2 p}, \log \frac{\phi_{i} g^{2 p}}{\sqrt{\lambda_{i}} g_{i} h_{\lambda}}\right\rangle+\sum_{i=1}^{n} \lambda_{i}\left\langle g_{i}^{2 p}, \log \frac{\phi_{i} \mathfrak{A}\left(h_{\lambda}\right)}{\sqrt{\lambda_{i}} g_{i}}\right\rangle\right] \\
\leq p & {\left[\sum_{i=1}^{n} \lambda_{i} \log \left\langle g_{i}^{2 p-1} \frac{\phi_{i}}{\sqrt{\lambda_{i}}}, \frac{g^{2 p}}{h_{\lambda}}\right\rangle+\sum_{i=1}^{n} \lambda_{i} \log \left\langle g_{i}^{2 p-1} \frac{\phi_{i}}{\sqrt{\lambda_{i}}}, \mathfrak{A}\left(h_{\lambda}\right)\right\rangle\right] } \\
\leq & p\left[\log \left\langle\sum_{i=1}^{n} \sqrt{\lambda_{i}} g_{i}^{2 p-1} \phi_{i}, \frac{g^{2 p}}{h_{\lambda}}\right\rangle+\log \left\langle h_{\lambda}, \mathfrak{A}\left(h_{\lambda}\right)\right\rangle\right] \\
= & p \log \left\|h_{\lambda}\right\|_{\mathrm{E}}^{2} .
\end{aligned}
$$

This shows that (4.11) holds and implies the upper bound in (4.10).

To prove the lower bound in (4.10) we pick, in accordance with Lemma 4.1 maximisers $\lambda \in \mathbb{S}_{n}$ and $g_{1}, \ldots, g_{n} \in L^{2 p}(B)$ for the problem of the right-hand side and show that the value of the functional of the left-hand side for the choice $\mu_{i}(d x)=g_{i}^{2 p}(x) d x$ for $i=1, \ldots, n$ is not smaller than the value of the maximum on the right. Recall from (4.4) that $\mu_{i}$-almost everywhere $\phi_{i}>0$. We first find an upper bound for $\mathcal{G}\left(\sum_{i=1}^{n} \lambda_{i} \mu_{i}\right)$ by picking some particular $\nu \in \mathcal{M}_{1}\left(U^{2}\right)$; recall the definition (2.5) of $\mathcal{G}$. Indeed, define $\nu^{*} \in \mathcal{M}_{1}\left(U^{2}\right)$ by

$$
\nu^{*}(d x d y)=\frac{1}{\rho} h_{\lambda}(x) G(x, y) h_{\lambda}(y) d x d y
$$

where we abbreviated $\rho=\rho(\phi)$ and $h_{\lambda}=\sum_{i=1}^{n} \sqrt{\lambda_{i}} g_{i}^{2 p-1} \phi_{i}$. Note from (4.4) that, for all $i=1, \ldots, n$,

$$
\mathfrak{A}\left(h_{\lambda}\right)(x)=\rho \sqrt{\lambda_{i}} \frac{g_{i}(x)}{\phi_{i}(x)}, \text { if } \phi_{i}(x)>0
$$

Note that $\nu^{*}$ is an admissible choice in the optimisation problem in the definition (2.5) of $\mathcal{G}$, by symmetry and because, using (4.12),

$$
\bar{\nu}^{*}(d y)=\frac{1}{\rho} h_{\lambda}(y) \mathfrak{A}\left(h_{\lambda}\right)(y) d y=\sum_{i=1}^{n} \lambda_{i} g_{i}^{2 p} d y=\mu(d y) .
$$


Replacing the supremum over all $\nu$ by the value for $\nu^{*}$ gives the following lower bound for the left-hand side of (4.10):

$$
\begin{aligned}
-\sum_{i=1}^{n} & \left\langle\lambda_{i} \mu_{i}, \log \left(\frac{\lambda_{i}^{p}}{\phi_{i}^{2 p}} \frac{d \mu}{d x}\right)\right\rangle-p \mathcal{G}\left(\sum_{i=1}^{n} \lambda_{i} \mu_{i}\right) \\
& =p\left[-\sum_{i=1}^{n} \lambda_{i}\left\langle\mu_{i}, \log \frac{\lambda_{i} g_{i}^{2}}{\phi_{i}^{2}}\right\rangle+\sup _{\nu: \bar{\nu}=\mu} \iint \nu(d x d y) \log \frac{g^{2 p}(x) G(x, y) g^{2 p}(y)}{\frac{\nu(d x d y)}{d x d y}}\right] \\
& \geq p\left[-\sum_{i=1}^{n} \lambda_{i}\left\langle\mu_{i}, \log \frac{\lambda_{i} g_{i}^{2}}{\phi_{i}^{2}}\right\rangle+\iint \nu^{*}(d x d y) \log \frac{g^{2 p}(x) G(x, y) g^{2 p}(y)}{\frac{\nu^{*}(d x d y)}{d x d y}}\right] \\
& =p\left[-2 \sum_{i=1}^{n} \lambda_{i}\left\langle\mu_{i}, \log \frac{\sqrt{\lambda_{i}} g_{i}}{\phi_{i}}\right\rangle+\log \rho+2 \int \mu(d x) \log \frac{g^{2 p}(x)}{h_{\lambda}(x)}\right] \\
& =p \log \left\|h_{\lambda}\right\|_{\mathrm{E}}^{2}=p \log \rho,
\end{aligned}
$$

because $\sqrt{\lambda_{i}} g_{i} h_{\lambda}=g^{2 p} \phi_{i}$ by (4.12) and the definition of $h_{\lambda}$. This completes the proof.

4.2. Identification of $\boldsymbol{W}(\phi)$ in terms of energies of functions. In this section, we identify the variational formula (4.2) for $\rho(\phi)$ in terms of the formula (1.7) for $\Theta(\phi)$ and prove Proposition 1.5 As a first step, we prove that minimisers exist in (1.7), and we derive their variational equation.

Lemma 4.3 (Analysis of $\Theta(\phi))$. Let $\phi=\left(\phi_{1}, \ldots, \phi_{n}\right)$ be a family of nonnegative, bounded measurable functions on $B$ having compact supports. Then there exists a $\psi \in \mathcal{D}(B)$, which satisfies

$$
\Theta(\phi)=\frac{p}{2}\|\nabla \psi\|_{2}^{2} \text { and } \sum_{i=1}^{n}\left\|\phi_{i} \psi\right\|_{2 p}^{2}=1,
$$

and with $h=\sum_{i=1}^{n}\left\|\phi_{i} \psi\right\|_{2 p}^{2-2 p} \phi_{i}^{2 p}$ we have the variational equations

$$
\frac{p}{\Theta(\phi)} \psi=\mathfrak{A}\left(\psi^{2 p-1} h\right) \quad \text { and } \quad-\frac{p}{2} \Delta \psi=\Theta(\phi) \psi^{2 p-1} h .
$$

Proof. As a first step, we derive the existence of a minimiser in (1.7). Let $\left(\psi_{k}\right.$ : $k \in \mathbb{N}$ ) be a minimising sequence, that is, the functions $\psi_{k} \in \mathcal{D}(B)$ are nonnegative and satisfy $\sum_{i=1}^{n}\left\|\phi_{i} \psi_{k}\right\|_{2 p}^{2}=1$ for any $k \in \mathbb{N}$, and $\lim _{k \uparrow \infty} \frac{1}{2}\left\|\nabla \psi_{k}\right\|_{2}^{2}=\Theta(\phi)$.

Let $\psi_{*} \in \mathcal{D}(B)$ denote the weak limit of a subsequence in accordance with Lemma 5.2. By local strong convergence in $L^{2 p}(B)$, we also have $\sum_{i=1}^{n}\left\|\phi_{i} \psi_{*}\right\|_{2 p}^{2}=$ 1. By weak lower semicontinuity of the map $\psi \mapsto\|\nabla \psi\|_{2}^{2}$ (apply [LL01, Theorem 2.11]), we have that $\frac{1}{2}\left\|\nabla \psi_{*}\right\|_{2}^{2} \leq \liminf _{k \uparrow \infty} \frac{1}{2}\left\|\nabla \psi_{k}\right\|_{2}^{2}=\Theta(\phi)$. Since $\psi_{*}$ is certainly nonnegative, it is a minimiser in (1.7).

The second step is the derivation of the variational equation in (4.13) for any minimiser $\psi_{*}$ in (1.7). Since $\|\nabla|\psi|\|_{2}^{2}=\|\nabla \psi\|_{2}^{2}$ for any $\psi \in \mathcal{D}(B)$ (see LL01, Theorem 6.17]), and since $\sum_{i=1}^{n}\left\|\phi_{i} \psi\right\|_{2 p}^{2}$ is positive homogeneous of order two in $\psi, \psi_{*}$ is also a minimiser in the variational problem

$$
\Theta(\phi)=\inf _{\psi \in \mathcal{D}(B)} \frac{\frac{p}{2}\|\nabla \psi\|_{2}^{2}}{\sum_{i=1}^{n}\left\|\phi_{i} \psi\right\|_{2 p}^{2}} .
$$

Denote the quotient on the right-hand side of (4.14) by $F(\psi)$. Let $\varphi \in C_{\mathrm{c}}^{\infty}(B)$ be a smooth test function. Then the map $\varepsilon \mapsto F\left(\psi_{*}+\varepsilon \varphi\right)$ can easily be differentiated 
at $\varepsilon=0$. By minimality of $\psi_{*}$ for $F$, this derivative is equal to zero. Recalling that $\sum_{i=1}^{n}\left\|\phi_{i} \psi_{*}\right\|_{2 p}^{2}=1$, this implies that

$$
\begin{aligned}
0 & =\left.\frac{d}{d \varepsilon}\right|_{\varepsilon=0}\left\|\nabla\left(\psi_{*}+\varepsilon \varphi\right)\right\|_{2}^{2}-\left.\sum_{i=1}^{n}\left\|\nabla \psi_{*}\right\|_{2}^{2} \frac{d}{d \varepsilon}\right|_{\varepsilon=0}\left\|\phi_{i}\left(\psi_{*}+\varepsilon \varphi\right)\right\|_{2 p}^{2} \\
& =2 \int_{B} \nabla \psi_{*} \cdot \nabla \varphi-4 \frac{\Theta(\phi)}{p} \sum_{i=1}^{n}\left\|\phi_{i} \psi_{*}\right\|_{2 p}^{2-2 p}\left\langle\varphi, \phi_{i}^{2 p} \psi_{*}^{2 p-1}\right\rangle \\
& =-2\left\langle\varphi, \Delta \psi_{*}+2 \frac{\Theta(\phi)}{p} \psi_{*}^{2 p-1} h\right\rangle,
\end{aligned}
$$

where we used the definition of the distributional Laplacian in the last step, and $h=\sum_{i=1}^{n}\left\|\phi_{i} \psi_{*}\right\|_{2 p}^{2-2 p} \phi_{i}^{2 p}$ as in 4.13). As (4.15) holds for any smooth test function $\varphi$, we infer that the function in the right argument of the brackets on the right-hand side is equal to zero, i.e., $-\frac{1}{2} \Delta \psi_{*}=\frac{\Theta(\phi)}{p} \psi_{*}^{2 p-1} h$, which is the second identity in (4.13). By [LL01, Th. 6.21], the function $\psi=\frac{\Theta(\phi)}{p} \mathfrak{A}\left(\psi_{*}^{2 p-1} h\right)$ satifies $-\frac{1}{2} \Delta \psi=$ $\frac{\Theta(\phi)}{p} \psi_{*}^{2 p-1} h$. Hence, by [LL01, Th. 9.3], $\psi$ differs from $\psi_{*}$ by a harmonic function in $\mathcal{D}(B)$, which therefore vanishes. This ends the proof of (4.13).

Now we identify $\rho(\phi)$ in terms of $\Theta(\phi)$. The following proposition completes the proof of Theorem 2.4, with the help of Proposition 2.2 and Proposition 4.2.

Proposition 4.4 (Relation between $\rho$ and $\Theta$ ). Let $\phi=\left(\phi_{1}, \ldots, \phi_{n}\right)$ be a family of nonnegative, bounded measurable functions on $B$ with compact supports. Then $\rho(\phi)=p / \Theta(\phi)$, i.e.,

$$
\begin{aligned}
\max \{\| & \left.\sum_{i=1}^{n} \sqrt{\lambda_{i}} g_{i}^{2 p-1} \phi_{i}\left\|_{\mathrm{E}}^{2}: \lambda \in \mathbb{S}_{n}, g_{i} \in L^{2 p}(B),\right\| g_{i} \|_{2 p}=1 \text { for } i=1, \ldots, n\right\}^{-1} \\
& =\min \left\{\frac{1}{2}\|\nabla \psi\|_{2}^{2}: \psi \in \mathcal{D}(B), \sum_{i=1}^{n}\left\|\phi_{i} \psi\right\|_{2 p}^{2}=1\right\}
\end{aligned}
$$

Remark 4.5. The proof gives an explicit one-to-one correspondence between the maximisers on the left and the minimisers on the right-hand side, see (4.17) and (4.19), respectively.

Proof. For the proofs of both ' $\geq$ ' and ' $\leq$ ' in (4.16), we pick the maximiser, resp. the minimiser, in one variational formula, construct admissible objects for the other one, and show that the other functional attains the inverse of the value of the maximum resp. minimum.

Let us begin with the proof of ' $\geq$ '. Pick maximisers $\lambda \in \mathbb{S}_{n}$ and $g_{1}, \ldots, g_{n} \in$ $L^{2 p}(B)$ of the formula on the right-hand side of formula (4.16) in accordance with Lemma 4.1. Define

$$
\psi=\frac{1}{\rho(\phi)} \mathfrak{A}\left(\sum_{j=1}^{n} \sqrt{\lambda_{j}} g_{j}^{2 p-1} \phi_{j}\right) .
$$

Then, by (4.4), for all $i=1, \ldots, n$,

$$
\psi(x)=\sqrt{\lambda_{i}} \frac{g_{i}}{\phi_{i}}(x) \text { for all } \phi_{i}(x)>0 .
$$


Hence,

$$
\sum_{i=1}^{n}\left\|\phi_{i} \psi\right\|_{2 p}^{2}=\sum_{i=1}^{n} \lambda_{i} \int g_{i}^{2 p}(x) d x=1 .
$$

Then the energy of the measure $\frac{1}{\rho(\phi)} \sum_{j=1}^{n} \sqrt{\lambda_{j}} g_{j}^{2 p-1} \phi_{j} d x$ can be calculated as follows:

$$
\begin{aligned}
\left\|\frac{1}{\rho(\phi)} \sum_{j=1}^{n} \sqrt{\lambda_{j}} g_{j}^{2 p-1} \phi_{j}\right\|_{\mathrm{E}}^{2} & =\frac{1}{\rho(\phi)} \int_{B} d x \psi(x) \sum_{j=1}^{n} \sqrt{\lambda_{j}} g_{j}^{2 p-1}(x) \phi_{j}(x) \\
& =\frac{1}{\rho(\phi)} \int_{B} d x \sum_{j=1}^{n} \lambda_{j} g_{j}^{2 p}(x)=\frac{1}{\rho(\phi)} .
\end{aligned}
$$

By Lemma 5.3 we have that $\psi \in \mathcal{D}(B)$ and that the energy of $\psi$ equals the energy of the measure $\frac{1}{\rho(\phi)} \sum_{j=1}^{n} \sqrt{\lambda_{j}} g_{j}^{2 p-1} \phi_{j} d x$, i.e., $\frac{1}{2}\|\nabla \psi\|_{2}^{2}=\frac{1}{\rho(\phi)}$. This implies ' $\geq$ '.

To prove ' $\leq$ ', we choose $\psi$ as the minimiser of the problem on the right-hand side of in (4.16), by Lemma 4.3. We define $g_{1}, \ldots, g_{n} \in L^{2 p}(B)$ and $\lambda \in \mathbb{S}_{n}$ by

$$
g_{i}=\frac{\psi \phi_{i}}{\left\|\psi \phi_{i}\right\|_{2 p}} \quad \text { and } \quad \lambda_{i}=\left\|\psi \phi_{i}\right\|_{2 p}^{2} \quad \text { for } i=1, \ldots, n .
$$

Note that $\left\|g_{i}\right\|_{2 p}=1$ for all $i=1, \ldots, n$ and that $\lambda_{1}, \ldots, \lambda_{n}$ are nonnegative numbers summing to one. Hence, $g_{1}, \ldots, g_{n}$ and $\lambda=\left(\lambda_{1}, \ldots, \lambda_{n}\right)$ are admissible for the formula on the left-hand side of (4.16). We find, using the first identity in (4.13),

$$
\begin{aligned}
& \left\|\sum_{i=1}^{n}{\sqrt{\lambda_{i}}}_{i}^{2 p-1} \phi_{i}\right\|_{\mathrm{E}}^{2}=\left\|\sum_{i=1}^{n}\right\| \psi \phi_{i}\left\|_{2 p}^{2-2 p} \psi^{2 p-1} \phi_{i}^{2 p}\right\|_{\mathrm{E}}^{2} \\
& =\sum_{j=1}^{n}\left\langle\psi^{2 p-1} \phi_{j}^{2 p}\left\|\psi \phi_{j}\right\|_{2 p}^{2-2 p}, \mathfrak{A}\left(\psi^{2 p-1} \sum_{i=1}^{n} \phi_{i}^{2 p}\left\|\psi \phi_{i}\right\|_{2 p}^{2-2 p}\right)\right\rangle \\
& =\frac{p}{\Theta(\phi)} \sum_{j=1}^{n}\left\langle\psi^{2 p-1} \phi_{j}^{2 p}\left\|\psi \phi_{j}\right\|_{2 p}^{2-2 p}, \psi\right\rangle=\frac{p}{\Theta(\phi)} .
\end{aligned}
$$

This completes the proof of the proposition.

\section{Appendix: The space $\mathcal{D}(B)$}

We now recall the definition of the function space $\mathcal{D}(B)$ and state some properties of this space. All of this material is known to the experts, but we find it convenient to collect some technical facts which are used at some places.

In the case of $B$ bounded, $\mathcal{D}(B)$ is the classical Sobolev space $H_{0}^{1}(B)$ which is defined as the closure of $\mathcal{C}_{\mathrm{c}}^{\infty}(B)$ in the sense of the Sobolev norm $\psi \mapsto$ $\left(\|\nabla \psi\|_{2}^{2}+\|\psi\|_{2}^{2}\right)^{1 / 2}$ in the Sobolev space $H^{1}(B)$. We first give a relation between $H_{0}^{1}(B)$ and $H^{1}\left(\mathbb{R}^{d}\right)$ in the case of a $C^{1}$-boundary.

Lemma 5.1. Let $B \subset \mathbb{R}^{d}$ be an open bounded set with $C^{1}$-boundary. Let $\psi \in$ $H^{1}\left(\mathbb{R}^{d}\right)$ such that $\psi=0$ a.e. on $B^{\mathrm{c}}$. Then the restriction of $\psi$ to $B$ lies in $H_{0}^{1}(B)$.

Proof. Our proof is an adaptation of the proof of Theorem 3 in [Ev98, Section 5.3.3]. First we pick, for any $\varepsilon>0$, a function $\varphi_{\varepsilon} \in \mathcal{C}^{\infty}\left(\mathbb{R}^{d}\right)$ such that $\varphi_{\varepsilon} \rightarrow \psi$ as $\varepsilon \downarrow 0$ in the Sobolev norm, and such that $\operatorname{supp}\left(\varphi_{\varepsilon}\right)$ is contained in the open $\varepsilon$-neighbourhood of $B$, which we denote by $B[\varepsilon]$. 
Fix $x^{0} \in \partial B$. Since $\partial B$ is $C^{1}$, there are $r>0$ and a $C^{1}$-function $\gamma: \mathbb{R}^{d-1} \rightarrow \mathbb{R}$ such that

$$
B \cap B\left(x^{0}, r\right)=\left\{x \in B\left(x^{0}, r\right): x_{1}<\gamma\left(x_{2}, \ldots, x_{d}\right)\right\} .
$$

Let $V=B \cap B\left(x^{0}, r / 2\right)$. For $\varepsilon>0$ define $\psi_{\varepsilon}: V \rightarrow \mathbb{R}$ by $\psi_{\varepsilon}(x)=\varphi_{\varepsilon}\left(x+\varepsilon \lambda \mathrm{e}_{1}\right)$ for $x \in V$, where $\mathrm{e}_{1}$ denotes the first unit vector and $\lambda>1$ is chosen such that $\operatorname{supp}\left(\psi_{\varepsilon}\right) \subset U$. Then $\psi_{\varepsilon} \in \mathcal{C}^{\infty}(V)$. Now the continuity of the $L^{2}$-norm under translations shows that $\lim _{\varepsilon \downarrow 0}\left\|\psi_{\varepsilon}-\widetilde{\psi}_{\varepsilon}\right\|_{H^{1}(V)}=0$. In particular, we have that $\psi_{\varepsilon} \rightarrow \psi$ in $H^{1}(V)$.

Now the rest of the proof is as in the proof of Theorem 3 in [Ev98, Section 5.3.3]. Indeed, using the compactness of $\partial B$, we find a finite covering of $\partial B$ with balls $B_{1}, \ldots, B_{N}$ in which $\partial B$ can be mapped differentiably onto a hyperplane. Within the ball $B_{i}$, we can approximate $\psi$ for any $i$ in $H^{1}$-norm by a $C^{\infty}$-function $\psi^{(i)}$ whose support lies within $B$. Extend the covering of $\partial B$ to a covering of $B$ by adding a suitable open set $B_{0}$ whose closure is contained in $B$. On $B_{0}$, we can approximate $\psi$ in $H^{1}$-norm by a $C^{\infty}$-function $\psi^{(0)}$ with support within $B$ (use Theorem 1 in [Ev98, Section 5.3.3]). Now pick a smooth partition of the unity $\left(\zeta_{i}: i=0, \ldots, N\right)$, subordinated to the covering $B_{0}, \ldots, B_{N}$ of $B$, and put $\varphi=\sum_{i=0}^{N} \psi^{(i)} \zeta_{i}$. It is then easily seen that $\varphi$ lies in $\mathcal{C}_{\mathrm{c}}^{\infty}(B)$ and approximates $\psi$ in $H^{1}(B)$-norm. This completes the proof.

In the case that $B=\mathbb{R}^{d}$, the space $\mathcal{D}\left(\mathbb{R}^{d}\right)=D^{1}\left(\mathbb{R}^{d}\right)$ is the space of functions $f \in L_{\text {loc }}^{1}\left(\mathbb{R}^{d}\right)$, which vanish at infinity, i.e., $\left\{x \in \mathbb{R}^{d}:|f(x)|>a\right\}$ has finite Lebesgue measure for any $a>0$, and whose distributional gradient is in $L^{2}\left(\mathbb{R}^{d}\right)$. Now we collect some sequential compactness properties of the space $\mathcal{D}(B)$.

Lemma 5.2. Suppose $\left(\psi_{k}\right)_{k \in \mathbb{N}}$ is a sequence in $\mathcal{D}(B)$ such that $\left(\left\|\nabla \psi_{k}\right\|_{2}\right)_{k \in \mathbb{N}}$ is bounded. Fix any $q \in(1,2 d /(d-2))$ for $d \geq 3$ and any $q>1$ for $d \leq 2$. Then there exists $\psi \in \mathcal{D}(B)$ and a subsequence $\left(\psi_{k_{j}}\right)_{j \in \mathbb{N}}$ such that $\nabla \psi_{k_{j}} \rightarrow \nabla \psi$ weakly in $L^{2}(B)$ and $\psi_{k_{j}} \rightarrow \psi$ locally strongly in $L^{q}(B)$.

Proof. Let us recall standard Sobolev inequalities; see [LL01, Theorems 8.3, 8.5]. There are positive constants $S_{d}$ for $d \geq 3$ and $S_{2, r}$ for $r>2$ such that

$$
\begin{aligned}
S_{d}\|\psi\|_{2 d /(d-2)}^{2} & \leq\|\nabla \psi\|_{2}^{2}, & & \text { for } d \geq 3, \psi \in D^{1}\left(\mathbb{R}^{d}\right), \\
S_{2, r}\|\psi\|_{r}^{2} & \leq\|\nabla \psi\|_{2}^{2}+\|\psi\|_{2}^{2}, & & \text { for } d=2, \psi \in H^{1}\left(\mathbb{R}^{d}\right), r>2 .
\end{aligned}
$$

We first consider the case $B=\mathbb{R}^{d}$. In particular, $d \geq 3$. Fix $1<q<2 d /(d-2)$ and apply Hölder's inequality and Sobolev's inequality to get, for any bounded measurable set $A \subset \mathbb{R}^{d}$ and any $\psi \in D^{1}\left(\mathbb{R}^{d}\right)$,

$$
\left\|\psi 1_{A}\right\|_{q} \leq\|\psi\|_{2 d /(d-2)} \operatorname{Leb}(A)^{\frac{2 d-d q+2 q}{2 d q}} \leq\|\nabla \psi\|_{2} S_{d}^{-1 / 2} \operatorname{Leb}(A)^{\frac{2 d-d q+2 q}{2 d q}},
$$

where Leb denotes the Lebesgue measure.

Now suppose that $\left(\psi_{k}\right)_{k \in \mathbb{N}}$ is a sequence in $D^{1}\left(\mathbb{R}^{d}\right)$ such that $\left(\left\|\nabla \psi_{k}\right\|_{2}\right)_{k \in \mathbb{N}}$ is bounded. The estimate in (5.2) shows that $\left(\psi_{k}\right)_{k \in \mathbb{N}}$ is locally bounded in $L^{q}\left(\mathbb{R}^{d}\right)$. By the Banach-Alaoglu Theorem, there is a subsequence $\left(\psi_{k_{j}}\right)_{j \in \mathbb{N}}$ which converges to some $\psi \in L^{q}\left(\mathbb{R}^{d}\right)$ locally weakly in $L^{q}\left(\mathbb{R}^{d}\right)$ and $\nabla \psi_{k_{j}} \rightarrow u$ weakly in $L^{2}\left(\mathbb{R}^{d}\right)$ for some $u \in L^{2}\left(\mathbb{R}^{d}\right)$. By [LL01, Theorem 8.6], the subsequence converges even locally strongly in $L^{q}\left(\mathbb{R}^{d}\right)$, and $u=\nabla \psi$. This completes the proof for $B=\mathbb{R}^{d}$.

Now we turn to the case of bounded $B$, for general $d \geq 1$. For any $\psi \in L^{2}(B)$ we define the extension $\psi_{*} \in L^{2}\left(\mathbb{R}^{d}\right)$ by $\psi_{*}(x)=0$ for $x \notin B$ and $\psi_{*}(x)=\psi(x)$ for 
$x \in B$. Then, for $\psi \in H_{0}^{1}(B)$ we have $\nabla \psi_{*}=(\nabla \psi)_{*}$. Indeed, let $\varphi \in C_{c}^{\infty}(B)$ be any test function and let $\psi_{n} \in C_{c}^{\infty}(B)$ be a sequence of functions approximating $\psi$ in the norm of $H_{0}^{1}(B)$. Then, applying partial integration to test functions, for all $1 \leq i \leq d$,

$$
\begin{aligned}
\int \varphi\left(\frac{\partial \psi}{\partial x_{j}}\right)_{*} & =\int_{B} \varphi \frac{\partial \psi}{\partial x_{j}}=\lim _{n \uparrow \infty} \int_{B} \varphi \frac{\partial \psi_{n}}{\partial x_{j}}=\lim _{n \uparrow \infty} \int_{B} \frac{\partial \varphi}{\partial x_{j}} \psi_{n} \\
& =\int_{B} \frac{\partial \varphi}{\partial x_{j}} \psi \cdot=\int \frac{\partial \varphi}{\partial x_{j}} \psi_{*} .
\end{aligned}
$$

This fact will be used in the sequel mostly without further notice. It implies, for instance, that Sobolev's inequality (5.1) is applicable to functions in $\mathcal{D}(B)=$ $H_{0}^{1}(B)$.

Suppose that $\left(\psi_{k}\right)_{k \in \mathbb{N}}$ is a sequence in $H_{0}^{1}(B)$ such that $\left(\left\|\nabla \psi_{k}\right\|_{2}\right)_{k \in \mathbb{N}}$ is bounded. In the case $d \geq 3$ and $1<q<2 d /(d-2)$, similarly to (5.2), one derives that $\left(\psi_{k}\right)_{k \in \mathbb{N}}$ is bounded in $L^{q}(B)$. By the Banach-Alaoglu theorem in the space $H_{0}^{1}(B)$, a subsequence converges weakly to some $\psi \in H_{0}^{1}(B)$, and the rest of the proof is as above in the case $B=\mathbb{R}^{d}$.

In the case $d \leq 2$, fixing any $q>1$, we first argue that there is a constant $C>0$ (depending only on $B$ and $q$ ) such that

$$
\|\psi\|_{q} \leq C\|\nabla \psi\|_{2}, \quad \text { for any } \psi \in H_{0}^{1}(B) .
$$

In order to prove (5.3) in $d=2$, use Hölder's inequality and the Sobolev inequality in (5.1) to obtain, for any $\psi \in H_{0}^{1}(B)$ and any $r>2$,

$$
\|\psi\|_{2}^{2} \leq\|\psi\|_{r}^{2} \operatorname{Leb}(B)^{1-2 / r} \leq \frac{\operatorname{Leb}(B)^{1-2 / r}}{S_{2, r}}\left(\|\nabla \psi\|_{2}^{2}+\|\psi\|_{2}^{2}\right) .
$$

It is known [LL01, Theorem 8.5] that $1 / S_{2, r}<\left(r^{2}(r-2) /[(r-1) 8 \pi]\right)^{1-2 / r} /(r-1)$. A Taylor approximation for $r \downarrow 2$ shows that the quotient on the right side of (5.4) is smaller than one for $r>2$ sufficiently close to 2. For this $r$, (5.4) can be solved for $\|\psi\|_{2}^{2}$, and we obtain the existence of a constant $c>0$ such that $\|\psi\|_{2}^{2} \leq c\|\nabla \psi\|_{2}^{2}$. Use this estimate on the right-hand side of (5.1) for $r=q$ to obtain that (5.3) holds for some $C>0$, only depending on $B$ and $q$.

In order to prove (5.3) in $d=1$, we use the simple inequality

$$
|f(x)|^{2} \leq\left\|f^{\prime}\right\|_{2}\|f\|_{2}, \quad \text { for } f \in H^{1}(\mathbb{R}), x \in \mathbb{R} ;
$$

see e.g. [LL01, Theorem 8.5(6)]. Now assume $q>2$. Raising (5.5) to the power $q / 2$ and integrating over $B$, we get, for $f \in H_{0}^{1}(B)$,

$$
\|f\|_{q}^{2} \leq\left\|f^{\prime}\right\|_{2}\|f\|_{2} \operatorname{Leb}(B)^{2 / q} \leq\left\|f^{\prime}\right\|_{2}\|f\|_{q} \operatorname{Leb}(B)^{1 / q+1 / 2}
$$

where we used Hölder's inequality in the second step. This shows (5.3) in the case $q>2$. The general case follows by a further application of Hölder's inequality.

The remainder of the proof in the case $d \leq 2$ is as above.

Recall the definition of the energy of a measure from (4.1). The following connection between the energy of functions in $\mathcal{D}(B)$ and the energy of measures will be important.

Lemma 5.3. For any (positive) absolutely continuous measure $\mu \in \mathcal{M}(B)$ whose support is a compact subset of $B$ and whose energy $\|\mu\|_{\mathrm{E}}^{2}$ is finite, the function $\psi=\mathfrak{A}(\mu)$ lies in $\mathcal{D}(B)$ and satisfies $\frac{1}{2}\|\nabla \psi\|_{2}^{2}=\|\mu\|_{\mathrm{E}}^{2}$. 
Proof. Let us look at bounded $B$ first. From (5.2) in the case $d \geq 3$ and from (5.3) in the case $d=2$ we get that for some $c>0$ we have $\|\nabla f\|_{2}^{2} \geq c\|f\|_{2}^{2}$, for all $f \in H_{0}^{1}(B)$. By [S98, Proposition 2.5.1], this coercivity condition implies that $\psi \in H_{0}^{1}(B)$ and $\frac{1}{2}\|\nabla \psi\|_{2}^{2}=\|\mu\|_{\mathrm{E}}^{2}$, as claimed.

Now suppose that $B=\mathbb{R}^{d}, d \geq 3$. Choose $n \in \mathbb{N}$ so large that the open centred ball $B(0, n)$ contains the support of $\mu$. The first part shows that the function $\psi_{n}=\mathfrak{A}_{n}(\mu)$, defined with the operator $\mathfrak{A}_{n}$ associated with the Green function $G_{n}$ on $B(0, n)$, lies in $H_{0}^{1}(B(0, n))$ and satisfies $\frac{1}{2}\left\|\nabla \psi_{n}\right\|_{2}^{2}=\|\mu\|_{\mathrm{E}, n}^{2}$, where the energy $\|\cdot\|_{\mathrm{E}, n}^{2}$ is taken with respect to the domain $B(0, n)$. We can extend each $\psi_{n}$ by zero to the whole of $\mathbb{R}^{n}$ and call the extension $\psi_{n}$ again. As $n \uparrow \infty$ we have $\psi_{n}=\mathfrak{A}_{n} \mu \uparrow \mathfrak{A} \mu=\psi$ and $\left\|\nabla \psi_{n}\right\|_{2}^{2}=\left\langle\mu, \mathfrak{A}_{n} \mu\right\rangle \uparrow\langle\mu, \mathfrak{A} \mu\rangle=\|\mu\|_{\mathrm{E}}^{2}$. From this, in combination with Lemma 5.2 , we see that $\psi \in D^{1}\left(\mathbb{R}^{d}\right)$.

Finally, we have to show that $\lim _{n \uparrow \infty}\left\|\nabla \psi_{n}\right\|_{2}=\|\nabla \psi\|_{2}$. For this, it is sufficient to show that $\lim _{n, m \uparrow \infty}\left\|\nabla\left(\psi_{n}-\psi_{m}\right)\right\|_{2}^{2}=0$. Use partial integration (see [LL01, Theorem 6.21]) and the facts that $-\frac{1}{2} \Delta \psi_{n}=\mu$ on $B(0, n)$ (as in [LL01, Theorem 6.21]) and $\psi_{m}=\mathfrak{A}_{m} \mu$ to see that, for any $n>m$,

$$
\begin{aligned}
\frac{1}{2}\left\|\nabla\left(\psi_{n}-\psi_{m}\right)\right\| & =\frac{1}{2}\left\|\nabla \psi_{n}\right\|_{2}^{2}+\frac{1}{2}\left\|\nabla \psi_{m}\right\|_{2}^{2}-\int_{B(0, n)} \nabla \psi_{n} \cdot \nabla \psi_{m} \\
& =\|\mu\|_{\mathrm{E}, n}^{2}+\|\mu\|_{\mathrm{E}, m}^{2}+\left\langle\Delta \psi_{n}, \psi_{m}\right\rangle \\
& =\left\langle\mu, \mathfrak{A}_{n} \mu\right\rangle+\left\langle\mu, \mathfrak{A}_{m} \mu\right\rangle-2\left\langle\mu, \mathfrak{A}_{m} \mu\right\rangle=\left\langle\mu,\left(\mathfrak{A}_{n}-\mathfrak{A}_{m}\right) \mu\right\rangle \\
& =\iint \mu(d x)\left(G_{n}(x, y)-G_{m}(x, y)\right) \mu(d y),
\end{aligned}
$$

where $G_{n}$ denotes Green function of $B(0, n)$. By Lebesgue's theorem, the righthand side vanishes as $n, m \uparrow \infty$.

\section{ACKNOWLEDGEMENT}

This work was supported in part by DFG grant contract number 234298, and by grant NAL/00631/G from the Nuffield foundation. The paper was finished when we were taking part in the programme "Interaction and Growth in Complex Stochastic Systems" at the Isaac Newton Institute, Cambridge. We would like to thank the Institute for their hospitality.

\section{REFERENCES}

[BS02] A.N. Borodin And P. SAlminen. Handbook of Brownian motion-facts and formulae. $2^{\text {nd }}$ edition. Birkhäuser, Basel (2002). MR1912205 (2003g:60001)

[Ch04] X. Chen. Exponential asymptotics and law of the iterated logarithm for intersection local times of random walks. Ann. Probab., 32, 3248-3300 (2004). MR2094445

[DZ98] A. Dembo And O. Zeitouni. Large deviations techniques and applications. $2^{\text {nd }}$ edition. Springer, New York (1998). MR.1619036 (99d:60030)

[Ev98] L.C. Evans. Partial differential equations. AMS Graduate Studies, Vol. 19 (1998). MR.1625845 (99e:35001)

[Fe48] R.J. Feynman. Space-time approach to nonrelativistic quantum mechanics. Rev. Mod. Phys. 20, 367-387 (1948). MR0026940 (10:224b)

[FP99] P.J. Fitzsimmons And J. Pitman. Kac's moment formula and the Feynman-Kac formula for additive functionals of a Markov process. Stoch. Process. Appl., 79, 117-134 (1999). MR 1670526 (2000a:60136)

[Ka49] M. KAC. On the distribution of certain Wiener functionals. Trans. Amer. Math. Soc. 65, 1-13 (1949). MR0027960(10:383b) 
[KM02] W. KÖNIG AND P. MÖRTERs, Brownian intersection local times: upper tail asymptotics and thick points, Ann. Probab. 30, 1605-1656 (2002). MR1944002 (2003m:60230)

[LG86] J.-F. Le GaLl. Sur la saucisse de Wiener et les points multiples du mouvement brownien. Ann. Probab. 14, 1219-1244 (1986). MR.0866344 (88e:60097)

[LG87] J.-F. Le Gall. The exact Hausdorff measure of Brownian multiple points I. In: Seminar on Stochastic Processes 1986, 107-137, Birkhäuser, Boston (1987). MR0902429 (89a:60188)

[LG89] J.-F. LE GaLl. The exact Hausdorff measure of Brownian multiple points II. In: Seminar on Stochastic Processes 1988, 193-197, Birkhäuser, Boston (1989). MR0990482 (90f:60139)

[LL01] E.H. Lieb AND M. Loss. Analysis. $2^{\text {nd }}$ edition. AMS Graduate Studies, Vol. 14 (2001). MR 1817225 (2001i:00001)

[Pi86] R. PINSKY. A spectral criterion for the finiteness or infiniteness of stopped FeynmanKac functionals of diffusion processes. Ann. Probab. 14, 1180-1187 (1986). MR0866341 (88f:60138)

[S98] A.-S. Sznitman. Brownian motion, obstacles and random media. Springer, Berlin (1998). MR:1717054 (2001h:60147)

Institut für Mathematik, Technische Universität Berlin, Strasse des 17. Juni 136, 10623 Berlin, Germany

E-mail address: koenig@math.tu-berlin.de

Current address: Mathematical Institute, University Leipzig, Augustusplatz 10/11, 04109 Leipzig, Germany

E-mail address: koenig@math.uni-leipzig.de

Department of Mathematical Sciences, University of Bath, Claverton Down, Bath BA2 7AY, United Kingdom

E-mail address: maspm@bath.ac.uk 